\title{
Time- and State-Dependent Input Delay-Compensated Bang-Bang Control of a Screw Extruder for 3D Printing
}

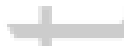 \\ Mamadou Diagne ${ }^{1 *}$ Nikolaos Bekiaris-Liberis $^{2}$ and Miroslav Krstic $^{3}$ \\ ${ }^{1}$ Department of Mechanical Engineering, University of Michigan, Ann Arbor, MI 48109-2102, USA. E-mail: \\ moliagne@umich.edu. \\ ${ }^{2}$ Department of Production Engineering and Management, Technical University of Crete, Chania, 73100, Greece. \\ E-mail: nikos.bekiaris@dssl.tuc.gr. \\ ${ }^{3}$ Department of Mechanical and Aerospace Engineering, University of California, San Diego, La Jolla, CA, 92093, \\ USA. E-mail: krsticeucsd.edu.
}

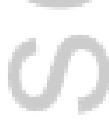

\section{SUMMARY}

In this paper a delay-compensated Bang-Bang control design methodology for the control of the nozzle output flow rate of screw extruder-based 3D printing processes is developed. A geometrical decomposition of the screw extruder in a partially and a fully filled regions allows to describe the material convection in the extruder chamber by a 1D hyperbolic Partial Differential Equation (PDE) coupled with an Ordinary Differential Equation (ODE). After solving the hyperbolic PDE by the Method of Characteristics (MC), the coupled PDE-ODE's system is transformed into a nonlinear state-dependent input delay system. The aforementioned delay system is extended to the non-isothermal case with the consideration of periodic fluctuations acting on the material's convection speed, which represent the process variabilities due to temperature changes in the extruder chamber, resulting to a nonlinear system with an input delay that simultaneously depends on the state and the time variable. Global Exponential Stability (GES) of the nonlinear delay-free plant is established under a piecewise exponential feedback controller that is designed. By combining the nominal, piecewise exponential feedback controller with nonlinear predictor feedback the compensation of the time- and state-dependent input delay of the extruder model is achieved. Global Asymptotic Stability (GAS) of the closed-loop system under the Bang-Bang predictor feedback control law is established when certain conditions related to the extruder design and the material properties, as well as to the magnitude and frequency of the materials transport speed variations, are satisfied. Simulations results are presented to illustrate the effectiveness of the proposed control design.

Copyright (c) 0000 John Wiley \& Sons, Ltd.

Received

KEY WORDS: Coupled PDE/ODE, time-varying delay, Bang Bang control, extrusion, 3D printing.

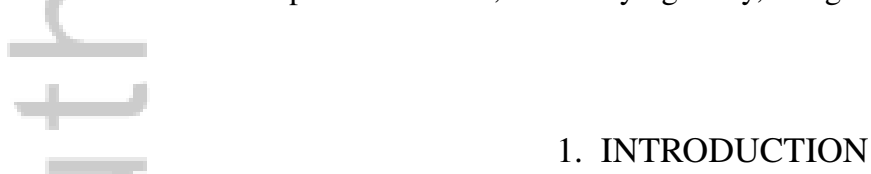

Additive Manufacturing (AM) has a promising future and demonstrates its effectiveness in various applications involving tissue engineering [4, 5], chemical engineering [6], thermoplastics [7], metal [8] and ceramic [9] material's fabrication. Functional 3D objects with complex geometrical shape can be produced in a short time without the needs of tools thanks to the Computer Aid Design This is the that drastically reduces the products development procedure Gurrently, the most popular but plastics 3D printers are based on FBM [18, 11, 12] and SBE [13, 7] technologies (Figure. 1).

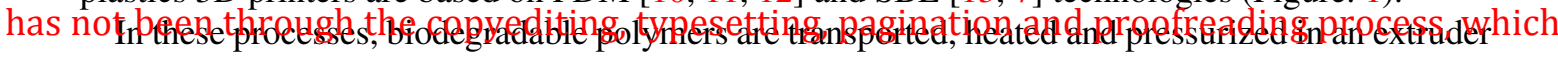

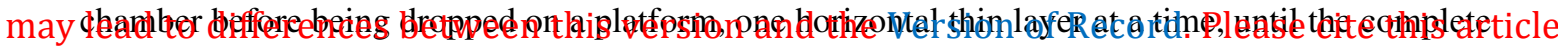
as doi: 10.1002/rnc.3761

${ }^{*}$ Correspondence to: Mamadou Diagne, e-mail: mdiagne@umich.edu.

Copyright (c) 0000 John Wiley \& Sons, Ltd.

Prepared using rncauth.cls [Version: 2010/03/27 v2.00]

This article is protected by copyright. All rights reserved. 
3D part is built such that it closely resembles the original CAD model. One of the crucial point that is not commonly addressed in the existing literature of extrusion-based 3D printing is controlling of the start and stop of extrusion-on-demand. A hybrid extrusion force-velocity modeling and tracking control for the fabrication of functionally graded material parts is developed in [14] using a first order differential equation that describes the plunger dynamic in a SBE process. Some extents of that approach are proposed by [15] with a robust tracking of the extrusion force to recover constant flow disturbances whereas [16] considers an unknown transfer function gain with an adaptive control strategy. Several issues regarding on FDM are discussed in $[17,18]$ and references therein, including the potential clogging due to agglomerate formation at the nozzle, appearance of bubbles, density inhomogeneity, tracking of short time-scale process variations, and prediction of anomalies such as material overflow and underflow for diverse applications. Thermal control is left out of most prior studies which are essentially based on empirical models.
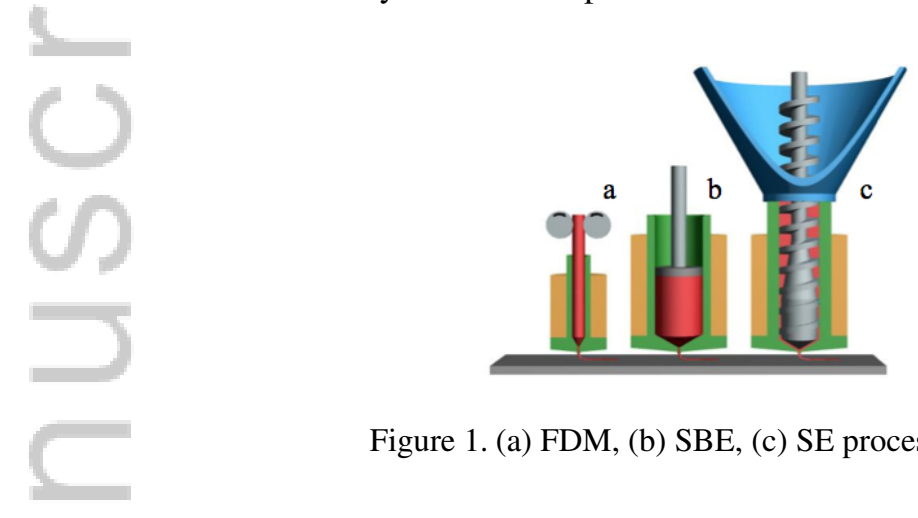

Figure 1. (a) FDM, (b) SBE, (c) SE processes [7].

In this paper, we are interested in the flow control issues related to the recent advances of 3D printing technology for which a Screw Extrusion (SE) process is utilized. In SE, the rotating screw allows a continuous feeding mechanism and generates a sufficiently high pressure in the extruder chamber, increasing, as a result, the printing speed. In addition, the screw motion extends the mixing capabilities of the system, and thereby, reduces drastically the risk of potential clogging at the nozzle while improving the homogeneity of the extruded filament [7, 19]. The SE process with granular material moves beyond the restrictions of FDM and does not require filament-shaped raw materials to operate. Consequently, it enables the processing of a broader range of raw materials and permits an easy recycling of wasted plastic during extrusion [7, 19, 20, 21, 22, 23]. In a recent review of melt extrusion additive manufacturing processes [24], the authors emphasize that the traditional extrusion manufacturing processes which use a granular or pelletized feedstock with a screw-type extruder is theoretically feasible, but no such mechanism is used in a commercially available AM (Additive Manufacturing system). In such processes, the need to control the start and stop of the extrusion process on demand calls for advanced control methodologies that are capable of enhancing the final product's quality in an industrial level. Even if experimental results demonstrate the effectiveness of SE [19], [7], the challenging control problems arising in such applications are actually poorly investigated.

In the present article, a generic and dynamical model of a homogeneous melt SE process derived from mass and momentum balance laws [25, 26, 27, 28, 29, 30, 31, 32, 33] is used for the design of a delay-compensated Bang-Bang controller which permits a fast and accurate control of the flow at the nozzle output. The model consists of a 1D Partial Differential Equation (PDE) that is defined on a time-varying spatial domain whose dynamics obeys to an Ordinary Differential Equation (ODE). The transformation of the coupled PDE-ODE system into a state-dependent input delay system, which describes the dynamics of the material convection in the extruder chamber, is achieved after solving the PDE by the Method of Characteristics (MC) [27, 29]. In order to also account for potential periodic fluctuations of the materials transport speed when processing granular pellets [34], due to the thermal energy that is supplied into the system from the heater of the extruder and the mechanical shearing effect by the rotation of the screw, the state-dependent input delay model is extended to a nonlinear system with an input delay that depends simultaneously on the state and 
the time variable (see [35, 36] and [37] for the treatment of systems with time- and state-dependent delays). The choice of the fluctuation model is motivated by several studies, in which, external distrubances entering the process from various sources and internal flow instabilities are identified to generate periodic oscillations in the process [38, 34, 39, 40, 41].

In [29], a delay-compensated Bang-Bang control law is developed for the control of the nozzle output flow rate of an isothermal screw extrusion process, achieving GES of the delay-free plant at any given setpoint. By combining the nominal, piecewise exponential feedback controller [29] with nonlinear predictor feedback, which is extended from the state-dependent input delay case [35] to the case in which the vector field and the delay function depend explicitly on time, the compensation of the time- and state-dependent input delay of the non-isothermal screw extrusion model is achieved. GAS of the closed-loop system under the delay-compensated Bang-Bang controller is established when certain conditions, related to the extruder design and the material properties, as well as to the periodic fluctuations, are satisfied. Several simulations results are presented including the case in which there is uncertainty in the value of the periodic variations of the material's transport speed.

In [36] the design is based only on a constant-delay predictor, and thus, it does not aim at compensating a time- and state-dependent delay, which, in contrast, is the case in the present paper. For this reason, also the stability analysis in [36] is performed differently since it aims at proving robustness of the constant-delay predictor to an unknown time and state-dependent delay rather than to prove stability of the predictor-feedback controller that compensates a known time- and state-dependent delay. (Note that, although the delay-compensating design for a time- and statedependent delay may be presented in [36], it is neither employed nor its stability properties are analyzed.)

This paper is organized as follows: The screw extruder mechanisms and the bi-zone model of the extruder consisting of the transport PDE coupled with the ODE for the moving interface is discussed in Section 2. In Section 3, the transformation of the coupled PDE-ODE system into a state-dependent input delay system by computing the PDEs solution by the MC is presented and it is then extended to a nonlinear system with a time- and state-dependent input delay. The control of the delay-free plant with a piecewise exponential Bang-Bang-like control law is described in Section 4. In Section 5, we design the predictor feedback control law for nonlinear systems with time- and state-dependent delay acting on the input. The application of the predictor feedback control law to the screw extruder model is presented in Section 6. The paper ends with simulations, including a discussion on the robustness properties of a state-dependent input delay compensator to timevarying perturbations acting on the vector field and the delay function, in Section 7.

\section{3D PRINTING BASED ON SINGLE-SCREW EXTRUDERS}

\subsection{Extrusion process description and structural decomposition of the extruder into a partially and a fully filled zone}

A screw extruder is divided into one or several conveying zones (transport zones), melting zones (for material fusion) and mixing zones in which the extruded melt is submitted to high pressure, before its eviction through the nozzle [42, 43, 44, 45, 46, 33, 25, 27, 28]. The net flow rate at the extruder nozzle depends mainly on the material flow in the longitidunal direction given by 1D heat and mass transport equations [47, 48]. Another particularity of these processes is that they can be divided in geometric regions which are partially and fully filled called PFZ and FFZ, respectively (Figure 2). The PFZ which is submitted to an atmospheric pressure is a conveying region and the flow in the FFZ is determined by the pressure gradient building-up in that region due to the nozzle resistance. These two zones are coupled by an interface which moves according to the volume of material accumulated in the FFZ. Basically, the moving interface is located at the point where the pressure gradient passes from zero to a non null value. 


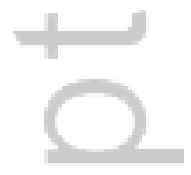

政

(

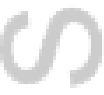

$+2$

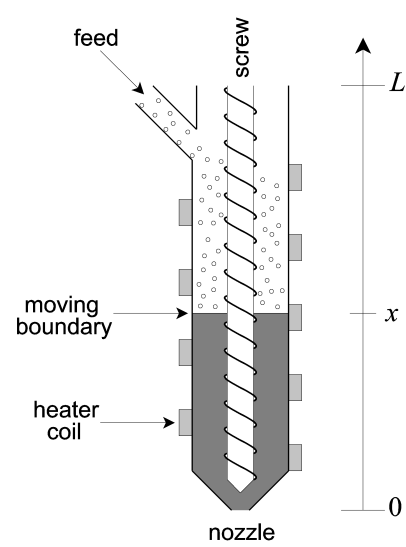

Figure 2. Bi-zone model of a screw extruder.

\subsection{Mass and momentum balance of an extrusion process}

2.2.1. Mass balance of the PFZ The PFZ is defined on the time-varying spatial interval $(x(t), L)$, $x(t)$, being the length of the FFZ and $L$ the extruder length (Figure 2). The mass balance in this area can be expressed using the fraction of the effective volume between a screw element and the barrel $\left(V_{\text {eff }}\right)$ which is occupied by the extruded material, namely, the filling ratio $u$. Considering an incompressible homogeneous mixture with constant density $\rho_{0}$ and viscosity $\eta$, the following mass conservation equation is deduced

$$
\begin{aligned}
\partial_{t} u(z, t) & =\xi N_{0} \partial_{z} u(z, t),(t, z) \in\left(\mathbb{R}^{+},(x(t), L)\right) \\
u(L, t) & =U(t),
\end{aligned}
$$

where $N_{0}$ is the constant screw speed and $\xi$ the uniform pitch of the screw. The boundary condition $u(L, t)$ is defined assuming the continuity of the flow at the inlet $\{z=L\}$

$$
U(t)=\frac{F_{\text {in }}(t)}{\rho_{0} N_{0} V_{\text {eff }}},
$$

where $F_{\text {in }}(t)$ is the feeding rate. Physically, the term $\rho_{0} N_{0} V_{\text {eff }}$ in (3) is the maximum pumping capacity of the screw.

2.2.2. Momentum balance of the FFZ The FFZ whose filling ratio is equal to one is defined on the spatial domain $(0, x(t))$, where the coordinate $\{z=0\}$ is the extruder's end. The FFZ flow depends on the pressure gradient that appears in this region, resulting to backward or forward flow. The momentum balance which is derived from Navier-Stokes equations under stationary conditions yields the pressure gradient

$$
\partial_{z} P(z, t)=-\eta \frac{\rho_{0} V_{\mathrm{eff}} N_{0}-F_{d}(t)}{B \rho_{0}},
$$

for all $(t, z) \in\left(\mathbb{R}^{+},(0, x(t))\right)$, where $B$ is a coefficient of pressure flow. The net flow rate $F_{d}(t)$, in the case of a Poiseuille flow is expressed with the help of the nozzle conductance $K_{d}$, the viscosity $\eta$, and the pressure at the nozzle $P(0, t)$ as

$$
\left\{\begin{array}{l}
F_{d}(t)=\frac{K_{d}}{\eta} \Delta P(t), \\
\Delta P(t)=P(0, t)-P_{0} .
\end{array}\right.
$$


2.2.3. Mass balance of the FFZ The FFZ mass balance leads to an ODE which describes the time evolution of its length. This length denoted by $x(t)$ determines the location of the small transfer region that is assimilated to the point at which the pressure changes from the atmospheric pressure $P_{0}$ to a different value $[45,46,25,49,28]$

$$
\frac{d x(t)}{d t}=\frac{\rho_{0} N_{0} V_{\mathrm{eff}} u(x(t), t)-F_{d}(t)}{\rho_{0} S_{\mathrm{eff}}(1-u(x(t), t))},
$$

where $S_{\text {eff }}$ is the available section and $V_{\text {eff }}=\xi S_{\text {eff }}$.

2.2.4. Coupling relations at the PFZ-FFZ interface The coupling condition is formulated imposing the pressure continuity at the spatial coordinate $x(t)$

$$
P\left(x^{-}, t\right)=P\left(x^{+}, t\right)=P_{0} .
$$

Integrating the pressure gradient equation (4), the net flow rate defined in (5) is written as

$$
F_{d}(t)=\frac{K_{d} V_{e f f} N_{0} \rho_{0} x(t)}{B \rho_{0}+K_{d} x(t)} .
$$

Substituting (5) in (6) and using (8), equation (6) for the length of the FFZ is written as

$$
\frac{d x(t)}{d t}=-\xi N_{0} \frac{K_{d} x(t)-\left(B \rho_{0}+K_{d} x(t)\right) u(x(t), t)}{\left(B \rho_{0}+K_{d} x(t)\right)(1-u(x(t), t))} .
$$

\section{FROM MASS BALANCE EQUATIONS OF THE EXTRUDER TO A DELAY SYSTEM}

\subsection{Isothermal delay system model}

The bi-zone model (1), (2), and (9) can be reduced to a nonlinear state dependent-input delay system [35]. The characteristic solutions of (1) with respect to the boundary condition (2) are

$$
u(z, t)=U\left(t-\frac{L-z}{\xi N_{0}}\right)
$$

Substituting (10) into (9), we derive the following nonlinear system

$$
\dot{x}(t)=\xi N_{0}\left[-\frac{K_{d} x(t)}{\left(B \rho_{0}+K_{d} x(t)\right)\left(1-U\left(t-D_{s}(x(t))\right)\right)}+\frac{U\left(t-D_{s}(x(t))\right)}{\left(1-U\left(t-D_{s}(x(t))\right)\right)}\right],
$$

for all $U(t) \in[0,1)$. The state-dependent input delay function is denoted as

$$
D_{s}(x(t))=\frac{L-x(t)}{\xi N_{0}} .
$$

A detailed derivation of the ODE (11) and an extensive description of the screw extruder model for $3 \mathrm{D}$ printing is given in [29].

\subsection{Delay system representation including process variabilities}

As stated in [41] the screw extrusion process always exibits complex and periodic fluctuations that arise from the effects of external distrubances entering the process from various sources, as well as internal flow instabilities ${ }^{\dagger}$. Internal instabilities such as cyclic breakup and buildup of the solid

\footnotetext{
$\dagger$ Under certain conditions, the amplitude of those fluctuations is large enough to cause undesirable effects such as surging or spurt flow, bamboo fracture, sharkskin, and other forms of product nonuniformity.
} 
bed in the melting, flow impurities, and nozzle flow instabilities are also well-known as potential sources of process variabilities [41].

Although the prediction of flow instability with good reliability for any given system has not been achieved so far, for specific applications several results state the oscillatory nature of extruders $[50,38,34,39,41]$. For instance, [41] insists on the periodic fluctuations that are damped out above a critical frequency with the expansion of the FFZ length and [34] uses a simplified model of an extrusion-cooker to study the effects of the low frequency periodic instability for a fluid whose viscosity is significantly reduced by the action of shear forces, considering a fully filled extruder and perturbing the constant flow velocity with a known sinusoidal signal. Other significant results include [40], which describes the process fluctuations using inferential monitoring of the screw load torque. Studying a virgin and recycled High-density Polyethylene (HPDE) material, [40] concludes that the periodic fluctuations occur at the screw speed frequency and are significantly higher for the virgin material than the recycled material at all screw speed except $10 \mathrm{rpm}$.

In this section, we account for such process variabilities, which might be provoked by temperature changes using the analytical framework developed in [34]. Specifically, knowing that the process is subject to oscillations we consider them as fluctuations acting on the convection speed of the PFZ. From the equivalence between the original PDE-ODE system (1)-(6) and the state-dependent input delay system (11), (12) we directly account for this model of fluctuations by modifying (11). We consider the following model in which the transport speed $c(t)$ is subjected to the periodic variabilities mentioned above.

$$
\dot{x}(t)=c(t)\left[-\frac{\theta_{2} x(t)}{\left(1+\theta_{2} x(t)\right)(1-U(t-D(t, x(t))))}+\frac{U(t-D(x(t)))}{(1-U(t-D(t, x(t))))}\right], U(t) \in[0,1),
$$
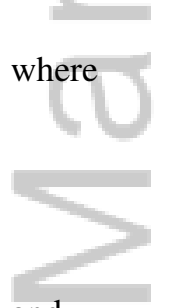

$$
\begin{aligned}
\theta_{1} & =\xi N_{0}, \\
\theta_{2} & =\frac{K_{d}}{B \rho_{0}},
\end{aligned}
$$

and,

$$
\begin{aligned}
D(t, x(t)) & =\frac{L-x(t)}{c(t)}, \\
c(t) & =\theta_{1}(1+\epsilon \cos (\omega t)),
\end{aligned}
$$

where, $0<\epsilon<1$ is a positive constant and $\omega$ is the mean value of the angular frequency of the periodic fluctuations. The restriction on $\epsilon$ is motivated by the fact that the PDE-ODE model (1)(6) is valid only when the convection speed is strictly positive and relaxing this contraint leads to a configuration in which the screw is pumping the material downwards, and thus the material is flowing in the opposite direction inside the barrel.

\section{CONTROL OF THE DELAY-FREE SYSTEM WITH A “BANG-BANG” CONTROL LAW}

\subsection{Review of the literature on screw extrusion control}

Due to the strong interaction between the mass, energy and momentum balances that occurs in screw extrusion processes, the design of efficient model-based controllers for such processes is still difficult to achieve. So far, most of the control-oriented models of extruders are derived from empirical and over-simplified models of limited operational range. Control strategies for control of the temperature profile, output flow rate, or pressure dynamics are proposed for traditional extrusion processes based on empirical models, including PID, predictive controllers and lead-lag compensators [51, 52, 54, 55]. 
To our knowledge the only controllers based on the bi-zone model (1)-(6) are proposed in [30],[31, 29, 27]. Particularly, in [30], a Lyapunov-based controller, based on the indirect measurement of the output pressure, achieves the stabilization of the output flow rate to a desired setpoint. A predictive controller is designed in [29], which, in fact, is the inspiration for the present paper. We mention that the exact controllability and the well-posedness of the bi-zone model (1)-(6) are also treated in [32].

\section{2. "Bang-Bang" controller design with piecewise exponential functions}

The starting point of the delay system controller design consists of the construction of a nonlinear control law that stabilizes the delay-free system

$$
\dot{x}(t)=c(t)\left[-\frac{\theta_{2} x(t)}{\left(1+\theta_{2} x(t)\right)(1-U(t))}+\frac{U(t)}{(1-U(t))}\right], \quad U(t) \in[0,1) .
$$

For $\epsilon<1$, the time-varying speed of the material transport $c(t)$ is strictly positive and the open-loop stabilizing control law of the delay-free plant (18) is given by

$$
v\left(x^{*}\right)=\frac{\theta_{2} x^{*}}{1+\theta_{2} x^{*}}, \quad \forall x^{*} \in[0, L) .
$$

This statement is directly derived considering the Lyapunov function $V=|e(t)|$, where $e(t)=$ $x(t)-x^{*}$.

For the feedback stabilization of (18), we consider two exponential functions [29]:

- for $x(t) \leq x^{*}$, a left-exponential function

$$
v_{1}\left(x, x^{*}\right)=v\left(x^{*}\right)+\left(v_{\max }-v\left(x^{*}\right)\right) \frac{1-e^{a_{1}\left(x^{*}\right)\left(x-x^{*}\right)}}{1-e^{-a_{1}\left(x^{*}\right)\left(x^{*}\right)}},
$$

where $a_{1}\left(x^{*}\right)>0$ is the gain of the left exponential control law. The function (20) takes values in $\left[v\left(x^{*}\right), v_{\max }\right]$, where $v_{\max }<1$ is the maximal value of the inlet filling ratio, namely the maximal feeding capacity of the extruder. Therefore, $v_{1}(0)=v_{\max }$, allows to set the inlet flow at its maximum capacity for a rapid refill action when the extruder is empty.

- for $x(t) \geq x^{*}$, a right-exponential function

$$
v_{\mathrm{r}}\left(x, x^{*}\right)=v\left(x^{*}\right)-v\left(x^{*}\right) \frac{1-e^{-a_{\mathrm{r}}\left(x^{*}\right)\left(x-x^{*}\right)}}{1-e^{-a_{\mathrm{r}}\left(x^{*}\right)\left(L-x^{*}\right)}},
$$

where $a_{\mathrm{r}}\left(x^{*}\right)>0$ is the gain of the right exponential control law. The function (21) belongs into the interval $\left[0, v\left(x^{*}\right)\right]$ and the control action stops radically the flow when the extruder is completely filled, namely, $v_{\mathrm{r}}(L)=0$.

\subsection{Extension of the "Bang-Bang" control law on the whole domain $(0, L)$}

Next, we introduce the characteristic function of the domains $\left[0, x^{*}\right]$ and $\left[x^{*}, L\right]$ and write the extended control law as

$$
v\left(x, x^{*}\right)=v_{\mathrm{l}}\left(x, x^{*}\right) h\left(x^{*}-x\right)+v_{\mathrm{r}}\left(x, x^{*}\right) h\left(x-x^{*}\right),
$$

where $h$ is the Heaviside function.

A continous slope function at the setpoint $x^{*}$ denoted by $S\left(x^{*}\right)$ is imposed to extend the left and the right exponential controllers (20) and (21), respectively into the differentiable piecewise exponential feedback law (22). The slope function is defined as $S(x)=-\frac{d v\left(x, x^{*}\right)}{d x}$ (the minus sign is conventional). More precisely, the key point of the design is to define a free parameter that may be specified by the user as the value of slope function at the equilibrium $S\left(x^{*}\right)$, under some restrictions that will be emphasized in this section. It is clear that, equiting the assigned value $S\left(x^{*}\right)$ to both left 
and right slope functions of (20) and (21), we can easily derive the following relations

$$
\begin{aligned}
S\left(x^{*}\right) & =\frac{a_{\mathrm{l}}\left(x^{*}\right)\left(v_{\max }-v\left(x^{*}\right)\right)}{1-e^{-a_{1}\left(x^{*}\right) x^{*}}}, \\
S\left(x^{*}\right) & =\frac{a_{\mathrm{r}}\left(x^{*}\right) v\left(x^{*}\right)}{1-e^{-a_{\mathrm{r}}\left(x^{*}\right)\left(L-x^{*}\right)}} .
\end{aligned}
$$

The equations (23) and (24) are both transcendental and admit numerical solutions namely the suitable exponential parameters needed to the left and to the right of the setpoint to achieve the differentiability of the controller (22). A unique solution $a_{1}\left(x^{*}\right)>0$ (respectively, $a_{\mathrm{r}}\left(x^{*}\right)>0$ ) exists if the linear and exponential functions of $a_{\mathrm{l}}\left(x^{*}\right)$ (respectively, $a_{\mathrm{r}}\left(x^{*}\right)$ ) have a strictly positive intersection (Figure 3), given by

$$
\left\{\begin{array}{l}
\psi_{1}\left(a_{1}\left(x^{*}\right)\right)=a_{1}\left(x^{*}\right)\left(v_{\max }-v\left(x^{*}\right)\right) \\
\phi_{1}\left(a_{1}\left(x^{*}\right)\right)=S\left(x^{*}\right)\left(1-e^{-a_{1}\left(x^{*}\right) x^{*}}\right) .
\end{array}\right.
$$

The sufficient condition, for a given $x^{*}$, for the given solution to (23) to be unique is

$$
\frac{d \psi_{\mathrm{l}}(0)}{d a_{\mathrm{l}}}<\frac{d \phi_{\mathrm{l}}(0)}{d a_{\mathrm{l}}}
$$

It follows that $S\left(x^{*}\right)$ should satisfiy

$$
S\left(x^{*}\right)>\frac{v_{\max }-v\left(x^{*}\right)}{x^{*}} .
$$

Similarly, we deduce that for a unique solution of (24) to exist (Figure 3), it must hold that

$$
\frac{d \psi_{\mathrm{r}}(0)}{d a_{\mathrm{r}}}<\frac{d \phi_{\mathrm{r}}(0)}{d a_{\mathrm{r}}}
$$

where

$$
\begin{cases}\psi_{\mathrm{r}}\left(a_{\mathrm{r}}\left(x^{*}\right)\right) & =a_{\mathrm{r}}\left(x^{*}\right) v\left(x^{*}\right) \\ \phi_{\mathrm{r}}\left(a_{\mathrm{r}}\left(x^{*}\right)\right) & =S\left(x^{*}\right)\left(1-e^{-a_{\mathrm{r}}\left(x^{*}\right)\left(L-x^{*}\right)}\right) .\end{cases}
$$

Hence

$$
S\left(x^{*}\right)>\frac{v\left(x^{*}\right)}{L-x^{*}}
$$

Finally, the minimal value of the setpoint slope $S_{\min }\left(x^{*}\right)$ above which there exist unique gains $a_{1}\left(x^{*}\right)$ and $a_{\mathrm{r}}\left(x^{*}\right)$ that ensure the differentiability of the extended control law $(22)$ on $(0, L)$ is given by

$$
S_{\min }\left(x^{*}\right)=\frac{1}{\frac{1}{\theta_{2} x^{*}}+1} \max \left\{\frac{v_{\max }\left(1+\frac{1}{\theta_{2} x^{*}}\right)-1}{x^{*}} ; \frac{1}{L-x^{*}}\right\}
$$

The speed of the controller or its "agressivness" increases with the rise of the setpoint slope $S\left(x^{*}\right)$. As it is illustrated in Figure 4, with the characteristics of the control law for the setpoints $x^{*}=0.02 \mathrm{~m}$ and $x^{*}=0.16 m$ with different values of the setpoint slope value $S\left(x^{*}\right)$.

\section{Theorem 1}

For any setpoint $x^{*} \in[0, L)$ and for any choosen setpoint slope $S\left(x^{*}\right) \in \mathbb{R}$ satisfying $S\left(x^{*}\right) \geq$ $S_{\min }\left(x^{*}\right)$, where $S_{\min }\left(x^{*}\right)$ is given by (31), taking the control gains $\left(a_{1}\left(x^{*}\right), a_{\mathrm{r}}\left(x^{*}\right)\right)$ as solutions of

$$
\begin{aligned}
a_{\mathrm{l}}\left(x^{*}\right)\left(v_{\max }-v\left(x^{*}\right)\right)-S\left(x^{*}\right)\left(1-e^{-a_{1}\left(x^{*}\right) x^{*}}\right) & =0, \\
a_{\mathrm{r}}\left(x^{*}\right) v\left(x^{*}\right)-S\left(x^{*}\right)\left(1-e^{-a_{\mathrm{r}}\left(x^{*}\right)\left(L-x^{*}\right)}\right) & =0 .
\end{aligned}
$$

The closed-loop system consisting of (18) with an initial condition $x_{0} \in[0, L)$ and the extended control law (20)-(22) is GES at $x=x^{*}$. 


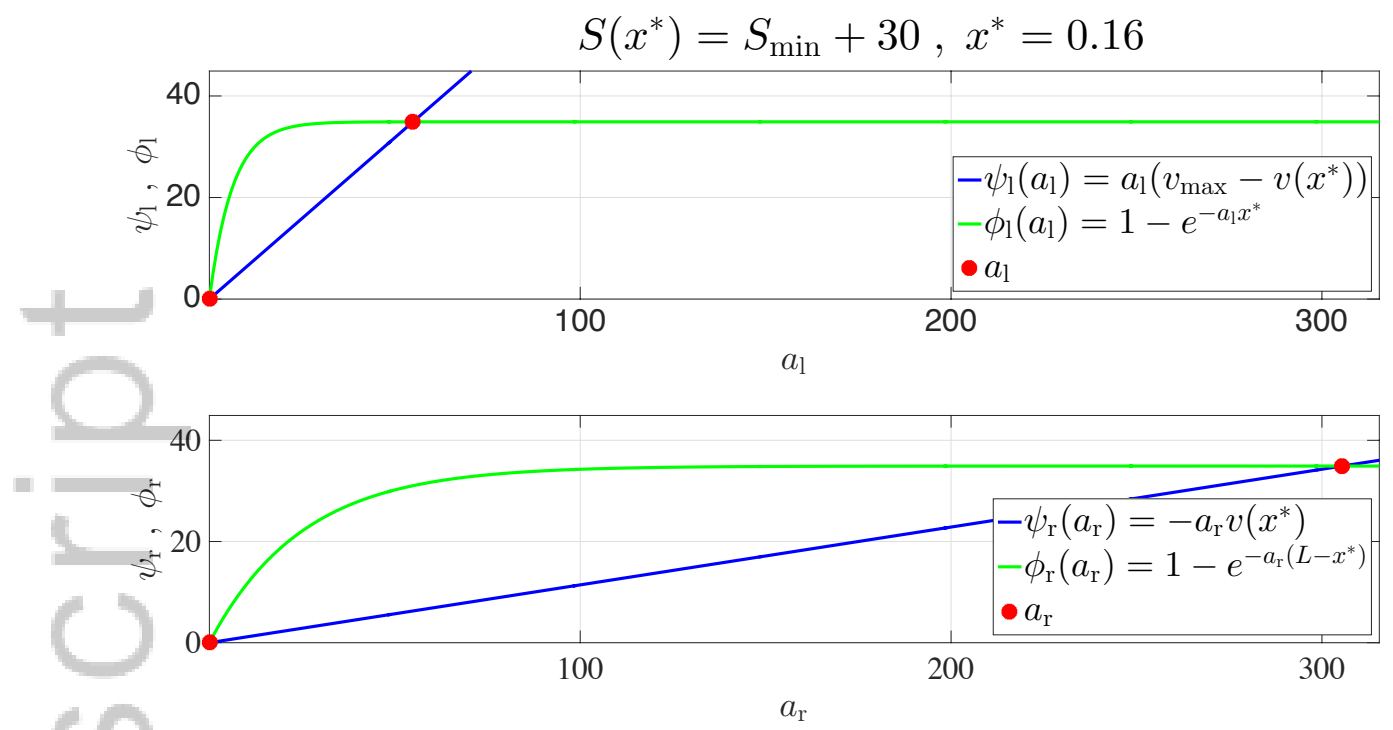

Figure 3. Gains $a_{\mathrm{l}}$ and $a_{\mathrm{r}}$ of the Bang-Bang controller.

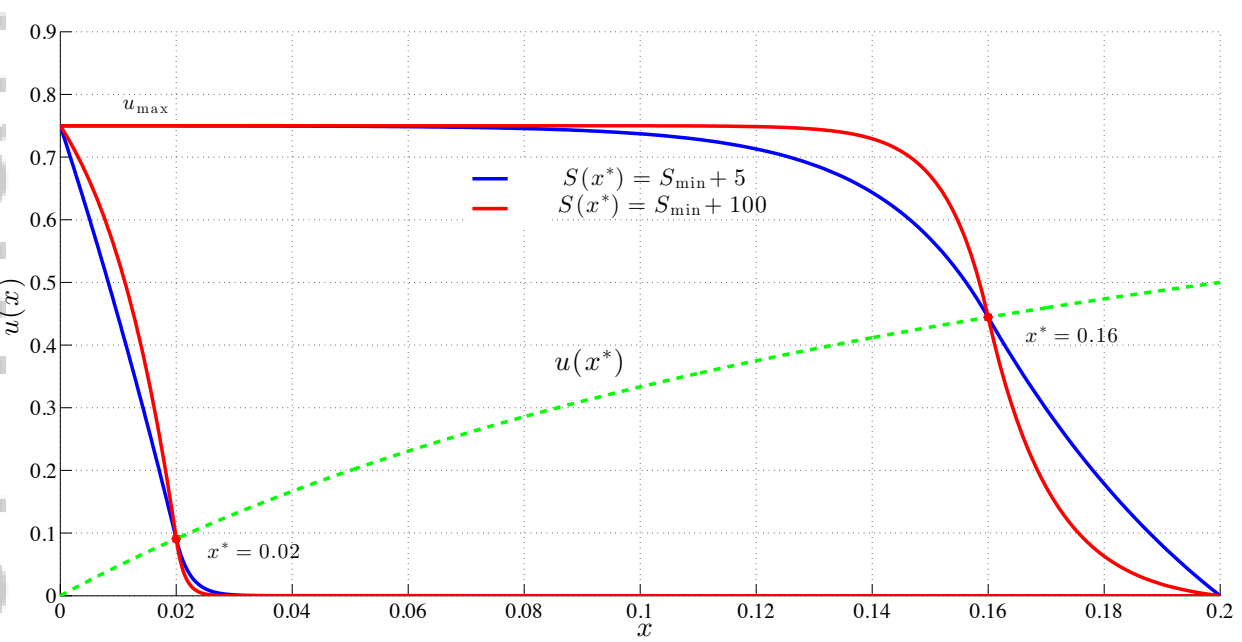

Figure 4. Control function for different $S_{\min }$ and setpoints.

Proof: We rewrite the delay-free plant (18) as

$$
\dot{e}(t)=\frac{c(t)\left(U(t)-v\left(x^{*}\right)\right)}{\left(1-v\left(x^{*}\right)\right)\left(1+\theta_{2}\left(e(t)+x^{*}\right)\right)(1-U(t))}-\frac{c(t) \theta_{2} e(t)}{1+\theta_{2}\left(e(t)+x^{*}\right)},
$$

where, $e(t)=x(t)-x^{*}$. The control law (22) is written as

$$
v\left(x(t), x^{*}\right)=v\left(e(t)+x^{*}, x^{*}\right) .
$$

The extended control law (22) is a decreasing function of $x$ and consequently for all $x(t) \in[0, L)$,

$$
\operatorname{sgn}\left(v\left(e(t)+x^{*}, x^{*}\right)-v\left(x^{*}, x^{*}\right)\right)=-\operatorname{sgn}(e(t)) .
$$


Moreover, $0 \leq v\left(x, x^{*}\right) \leq v_{\max }<1$ and $v\left(x^{*}, x^{*}\right)=v\left(x^{*}\right)$ is defined as the setpoint open-loop control (19). Next, we introduce the following Lyapunov function

$$
V=|e(t)| \text {. }
$$

Hence,

$$
\dot{V}=\dot{e}(t) \operatorname{sgn}(e(t)),
$$

and with the help of (36), by choosing $U(t)=v\left(x(t), x^{*}\right)$, (38) is written as

$$
\dot{V}=-\alpha(t) V-\beta(t),
$$

where $\alpha(t)>0$ and $\beta(t) \geq 0$ for all $x \in[0, L)$ and $U \in[0,1)$. The functions $\alpha(t)$ and $\beta(t)$ are given by

$$
\begin{aligned}
\alpha(t) & =\frac{c(t) \theta_{2}}{1+\theta_{2}\left(e(t)+x^{*}\right)} \\
\beta(t) & =\frac{1}{\left(1-v\left(e(t)+x^{*}, x^{*}\right)\right)} \times \frac{c(t)\left|v\left(e(t)+x^{*}, x^{*}\right)-v\left(x^{*}\right)\right|}{\left(1-v\left(x^{*}\right)\right)\left(1+\theta_{2}\left(e(t)+x^{*}\right)\right)},
\end{aligned}
$$

where, $c(t) \geq \theta_{1}(1-\epsilon)$, for all $t \geq 0$. Therefore

$$
\dot{V} \leq-\frac{\theta_{1}(1-\epsilon)}{1+\theta_{2} L} V .
$$

From (42) the closed-loop system is exponentially stable at $x^{*} \in[0, L)$ for all $x_{0} \in[0, L)$.

\section{PREDICTOR FEEDBACK CONTROL FOR NONLINEAR SYSTEMS WITH TIME- AND STATE-DEPENDENT INPUT DELAY}

\subsection{Predictor feedback design}

We consider the following nonlinear system with a time- and state-dependent input delay

$$
\begin{aligned}
\dot{x}(t) & =f(t, x(t), U(\phi(t))) \\
\phi(t) & =t-D(t, x(t)),
\end{aligned}
$$

where $x \in \mathbb{R}^{n}, U:\left[\phi\left(t_{0}\right), \infty\right) \rightarrow \mathbb{R}, t \geq t_{0} \geq 0, D \in C^{1}\left(\mathbb{R}_{+} \times \mathbb{R}^{n} ; \mathbb{R}_{+}\right)$, and $f: R_{+} \times \mathbb{R}^{n} \times \mathbb{R} \rightarrow$ $\mathbb{R}^{n}$ is locally Lipschitz with $f(t, 0,0)=0$ for all $t \geq 0$, and there exists a class $\mathcal{K}_{\infty}$ function $\hat{\alpha}$ such that

$$
|f(t, x, U)| \leq \hat{\alpha}(|x|+|U|) .
$$

Let us define $\kappa(t, x)$ as the nominal stabilizing feedback control law for the delay free plant $\dot{x}(t)=f(t, x(t), U(t))$. The predictor feedback control law for system (43), (44) is

$$
U(t)=\kappa(\sigma(t), P(t)),
$$

where, for all $\phi(t) \leq \theta \leq t$

$$
\begin{aligned}
& P(\theta)=x(t)+\int_{\phi(t)}^{\theta} \frac{f(\sigma(s), P(s), U(s))}{1-F(\sigma(s), P(s), U(s))} d s, \\
& \sigma(\theta)=t+\int_{\phi(t)}^{\theta} \frac{1}{1-F(\sigma(s), P(s), U(s))} d s,
\end{aligned}
$$

\footnotetext{
$¥$ As it is illustrated in Sections 2, and 3 such systems may derived from transport equations of the form (1) evolving on time-varying domains. Other examples of transport equations evolving on time-varying domains can be found in [56]. 
and

$$
F(\sigma(\theta), P(\theta), U(\theta))=\frac{\partial D}{\partial t}(\sigma(\theta), P(\theta))+\frac{\partial D}{\partial x}(\sigma(\theta), P(\theta)) \times f(\sigma(\theta), P(\theta), U(\theta)) .
$$

When simulating the predictor feedback controller (46)-(48), at each time step the ODE for the system (43)-(44) must be solved (using, for example, a simple Euler scheme) and the length of the delay must be computed (for example as the integer part of $N(i)=\frac{D(i, x(i))}{\tau}$, say $\bar{N}(i)$, where $\tau$ is the discretization step). The predictor is then computed by integrating simultateously the two integral relations (47) and (48) at each time step, using a numerical integration scheme. For instance, with the left endpoint rule of integration we get

$$
\begin{array}{r}
P(i)=x(i)+\tau \sum_{k=i-\bar{N}(i)}^{k=i-1} \frac{f(\sigma(k), P(k), U(k))}{1-F(\sigma(k), P(k), U(k))}, \\
\sigma(i)=i+\tau \sum_{k=i-\bar{N}(i)}^{k=i-1} \frac{1}{(1-F(\sigma(k), P(k), U(k))} .
\end{array}
$$

The prediction of the state at the time when the current control will have an effect on the state is defined as

$$
P(t)=x(t+D(\sigma(t), P(t))),
$$

where the prediction time is defined as

$$
\sigma(t)=t+D(\sigma(t), P(t))
$$

which is derived from the inversion of the time variable $t \rightarrow t-D(t, x(t))$ in $t \rightarrow t+$ $D(\sigma(t), P(t))$. Differentiating (52), (53) and using (43) we arrive at

$$
\frac{d x(\sigma(t))}{d t}=f(\sigma(t), x(\sigma(t)), U(t)) \frac{d \sigma(t)}{d t},
$$

and

$$
\dot{\sigma}(t)=\frac{1}{1-F(\sigma(t), P(t), U(t))}
$$

where $F$ is defined in (49). Finally, the implicit integral relations (47) and (48) are derived by integrating (54) and (55) on the delay interval $[\phi(t), \theta]$.

The key point of the predictor feedback design is the feasibility condition defined as

$$
\mathcal{F}_{c}: \quad \frac{\partial D}{\partial t}(\sigma(\theta), P(\theta))+\frac{\partial D}{\partial x}(\sigma(\theta), P(\theta)) f(\sigma(\theta), P(\theta), U(\theta))<c,
$$

for all $\theta \geq t_{0}-D\left(t_{0}, x\left(t_{0}\right)\right)$ and some $c \in(0,1)$. Condition (56) guarantees that the feedback control action can reach the plant, namely, the delay rate is bounded by unity, and that the denominator of the predictor (47) and the prediction time (48) is positive. We refer the reader to [35] for details on the predictor feedback control design and analysis for systems with state-dependent input delay.

\subsection{Stability analysis}

\section{Assumption 1}

There exist a continuously differentiable function $R$, class $\mathcal{K}_{\infty}$ functions $\mu_{1}, \mu_{2}, \mu_{3}$, and a positive constant $\chi$ such that for the plant $\dot{x}=f(t, x, \omega)$, the following hold

$$
\begin{gathered}
\mu_{1}(|x|) \leq R(t, x) \leq \mu_{2}(|x|) \\
\frac{\partial R(t, x)}{\partial t}+\frac{\partial R(t, x)}{\partial x} f(t, x, \omega) \leq \chi R(t, x)+\mu_{3}(|\omega|),
\end{gathered}
$$

for all $(x, \omega)^{T} \in \mathbb{R}^{n+1}$ and $t \geq t_{0}$. 
Assumption 1 guarantees that system $\dot{x}=f(t, x, \omega)$ is strongly forward complete with respect to $\omega$.

\section{Assumption 2}

There exist a locally Lipschitz function $\kappa \in\left(\left[t_{0}, \infty\right) \times \mathbb{R}^{n} ; \mathbb{R}\right)$ and a function $\hat{\rho} \in \mathcal{K}_{\infty}$ such that the system plant $\dot{x}=f(t, x, \kappa(t, x)+\omega)$ is input-to-state stable (ISS) with respect to $\omega$, with $\dot{x}=f(t, x, \kappa(t, x)+\omega)$ periodic with respect to $t$, and $\kappa$ is uniformly bounded with respect to its first argument, that is,

$$
|\kappa(t, x)| \leq \hat{\rho}(|x|) \quad \forall \quad t \geq t_{0} .
$$

Assumption 3

$D \in C^{1}\left(R_{+} \times R^{n} ; R_{+}\right), \frac{\partial D}{\partial t}$ and $\frac{\partial D}{\partial x}$ are locally Lipschitz (to guarantee the uniqueness of solutions), and there exist class $\mathcal{K}_{\infty}$ functions $\mu_{4}, \mu_{5}, \mu_{6}$ and non-negative constants $c_{1}, c_{2}, c_{3}$, with $c_{3}<c$, for some $0<c<1$, such that

$$
\begin{aligned}
D(t, x(t)) & \leq c_{1}+\mu_{4}(|x|), \\
\left|\frac{\partial D}{\partial x}(t, x(t))\right| & \leq c_{2}+\mu_{6}(|x|), \\
\left|\frac{\partial D}{\partial t}(t, x(t))\right| & \leq c_{3}+\mu_{5}(|x|) .
\end{aligned}
$$

The definitions of strong forward completeness and input-to-state stability are those from [57], and [58], respectively.

\section{Theorem 2}

Consider the closed-loop system consisting of the plant (43) and the control law (46)-(48). Under Assumptions 1, 2 and 3, there exist a class $\mathcal{K}$ function $\psi_{\text {RoA }}$ and a class $\mathcal{K} \mathcal{L}$ function $\beta_{s}$ such that for all initial conditions for which $U$ is locally Lipschitz on the interval $\left[t_{0}-D\left(t_{0}, x\left(t_{0}\right)\right), t_{0}\right)$ and which satisfy

$$
\Omega\left(t_{0}\right)<\psi_{\mathrm{RoA}}\left(c-c_{3}\right)
$$

for some $0<c<1$, where

$$
\Omega(t)=|x(t)|+\sup _{t-D(t, x(t)) \leq \theta \leq t}|U(\theta)|,
$$

there exists a unique solution to the closed-loop system with $x$ Lipschitz on $\left[t_{0}, \infty\right), U$ Lipschitz on $\left(t_{0}, \infty\right)$, and the following holds

$$
\Omega(t) \leq \beta_{s}\left(\Omega\left(t_{0}\right), t-t_{0}\right),
$$

for all $t \geq t_{0}$. Furthermore, there exists a positive constant $\gamma$ such that for all $t \geq t_{0}$,

$$
\begin{aligned}
D(t, x(t)) & \leq \gamma \\
\left|\frac{d D(t, x(t))}{d t}\right| & \leq c
\end{aligned}
$$

Proof of Theorem 2: Estimates (65), (66), and (67) follow by Lemmas 1-8 (see the Appendix). Existence and uniqueness of a solution $x$ Lipschitz on $[0, \infty)$ follows from the proof of Theorem 1 in [35] (page 7). It remains to show that $U$ is Lipschitz on $\left(t_{0}, \infty\right)$. Since $U(t)=\kappa(\sigma(t), P(t))$ and

$$
\begin{aligned}
\dot{P}(t) & =\frac{f(\sigma(t), P(t), \kappa(\sigma(t), P(t)))}{1-F(\sigma(t), P(t), \kappa(\sigma(t), P(t)))}, \\
\dot{\sigma}(t) & =\frac{1}{1-F(\sigma(t), P(t), \kappa(\sigma(t), P(t)))}, \\
F(\sigma(t), P(t)) & \left.=\frac{\partial D}{\partial x} D(\sigma(t), P(t)) f(\sigma(t), P(t), \kappa(\sigma(t), P(t)))\right)+\frac{\partial D}{\partial t}(\sigma(t), P(t)),
\end{aligned}
$$

for $t \geq t_{0}$, the Lipschitzness of $\frac{\partial D}{\partial t}, \frac{\partial D}{\partial x}, \kappa$ and $f$, and (56) ensure that the right hand-side of (68) and (69) are Lipschitz and consequently $(P, \sigma) \in\left(C^{1}\left(t_{0}, \infty\right) \times C^{1}\left(t_{0}, \infty\right)\right)$. From the Lipschitzness of $\kappa$, it follows that $U$ is Lipschitz. 


\section{APPLICATION TO THE EXTRUSION PROCESS MODEL}

From now, we recall the predictor feedback (46)-(48) for the compensation of the time- and statedependent input delay in system (13) that we rewrite formally as

$$
\begin{aligned}
\dot{x}(t) & =f(t, x(t), U(t-D(t, x(t)))), \\
f(t, x(t), U(t)) & =-c(t) \Gamma(x(t), U(t)),
\end{aligned}
$$

where $D(t, x(t))$ and $c(t)$ are defined in (16) and (17), respectively, and for all $U(t) \in[0,1)$

$$
\Gamma(x(t), U(t))=\frac{\theta_{2} x(t)}{\left(1+\theta_{2} x(t)\right)(1-U(t))}-\frac{U(t)}{1-U(t)},
$$

6.1. Verification of Assumptions 1, 2 and (3) for the screw extrusion model

- Assumption 1: From the definitions of the functions $\Gamma$ in (73) and $f$ expressed in terms of the error variable (34), we deduce from (17) that for all $0 \leq U \leq U_{m}$, with any $U_{m}<1$, and for all $x \in[0, \infty), 0<\epsilon<1, R(t, e)=\frac{e^{2}}{2}$ satisfies

$$
\frac{\partial R(t, e)}{\partial e} f_{e}(t, e, \tilde{U}) \leq \frac{\theta_{1}(1+\epsilon)|\tilde{U}||e|}{\left(1-v\left(x^{*}\right)\right)\left(1-U_{m}\right)} .
$$

where

$$
\left.f_{e}(t, e, \tilde{U})=f\left(t, e+x^{*}, \tilde{U}+v\left(x^{*}\right)\right)\right)
$$

and

$$
\tilde{U}=U-v\left(x^{*}\right)
$$

Using Young's inequality we arrive at

$$
\frac{\partial R(t, e)}{\partial e} f_{e}(t, e, \tilde{U}) \leq \frac{e^{2}}{2}+\frac{\theta_{1}^{2}(1+\epsilon)^{2}}{2\left(1-v\left(x^{*}\right)\right)^{2}\left(1-U_{m}\right)^{2}} \tilde{U}^{2} .
$$

From (77), choosing $R(t, e)=\frac{e^{2}}{2}$ and $\mu_{3}(\tilde{U})=\frac{(1+\epsilon)^{2}}{2\left(1-v\left(x^{*}\right)\right)^{2}\left(1-U_{m}\right)^{2}} \tilde{U}^{2}$, Assumption 1 is verified.

- Assumption 2: Taking $\kappa(t, x)=U\left(x, x^{*}\right)-v\left(x^{*}\right)$, where, $v\left(x, x^{*}\right)$ is defined in (22), the existence of a class $K_{\infty}$ function $\hat{\rho}$ such that (59) holds, follows given that $v\left(x, x^{*}\right) \in C^{1}[0, L]$. Setting $U=v\left(x, x^{*}\right)+\omega$ and using (34) and (37) we deduce that for all $\omega \leq 1-\omega_{m}$, for any $\nu_{m}$ such that $\omega_{m}>v_{\max }$, the following holds

$$
\begin{aligned}
\dot{V}(t) & \leq-\lambda V(t)+\frac{c(t)|\omega| \operatorname{sgn}(e(t))}{\Delta\left(x^{*}, e(t), \omega\right)} \\
\Delta\left(x^{*}, e(t), \omega\right) & =\left(1-v\left(x^{*}\right)\right)\left(1+\theta_{2}\left(e(t)+x^{*}\right)\right)\left(1-v\left(e(t)+x^{*}, x^{*}\right)-\omega\right) \\
\lambda & =\frac{\theta_{1}(1-\epsilon)}{1+\theta_{2} L}
\end{aligned}
$$

That is,

$$
\dot{V}(t) \leq-\lambda V(t)+\frac{2 \theta_{1}|\omega|}{\min \left|\Delta\left(x^{*}, e(t), \omega\right)\right|},
$$

and equivalently for $v\left(e(t)+x^{*}, x^{*}\right) \in\left[0, v_{\max }\right]$,

$$
\dot{V} \leq-\lambda V+\frac{2 \theta_{1}|\omega|}{\left(1-v\left(x^{*}\right)\right)\left(\omega_{m}-v_{\max }\right)} .
$$


Defining $\alpha(\omega) \in \mathcal{K}_{\infty}$, such that

$$
\alpha(\omega)=\frac{2 \theta_{1}|\omega|}{\left(1-v\left(x^{*}\right)\right)\left(\omega_{m}-v_{\max }\right)},
$$

from (82), we have

$$
\dot{V}(t) \leq-\lambda V(t)+\alpha(\omega) .
$$

Finally, from (84), Assumption 2 holds.

Assumption 3: From the delay function (16) and the fact that $0<\epsilon<1$, we get that $\min \{c(t)\}=\theta_{1}(1-\epsilon)$, and hence,

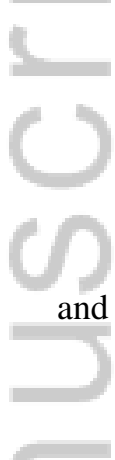

$$
\begin{gathered}
D(t, x)=\frac{L-x}{c(t)} \leq \frac{L}{\theta_{1}(1-\epsilon)} \\
\left|\frac{\partial D}{\partial x}(t, x)\right|=\frac{1}{c(t)} \leq \frac{1}{\theta_{1}(1-\epsilon)}
\end{gathered}
$$

Using (85), (86), and (87), it is easy to verify that conditions (60), (61), and (62) of Assumption 3 hold.

\subsection{Predictor feedback for the screw extrusion model}

The predictive feedback controller based on the piecewise exponential feedback law (22) is given by

$$
\begin{aligned}
& U(t)=v(P(t)), \\
& P(\theta)=x(t)+\int_{t-D(t, x(t))}^{\theta} \frac{f(\sigma(s), P(s), U(s))}{1-F(\sigma(s), P(s), U(s))} d s \\
& \sigma(\theta)=t+\int_{t-D(t, x(t))}^{\theta} \frac{1}{1-F(\sigma(s), P(s), U(s))} d s,
\end{aligned}
$$

for all $t-D(t, x(t)) \leq \theta \leq t$. The function $F$ defined in (49) for the system (71)-(72) is computed with the help of (16)-(17) as

$$
\begin{aligned}
F(\sigma(t), P(t), U(t))= & \frac{\theta_{1} \epsilon \omega \sin (\omega \sigma(t))(L-P(t))}{c^{2}(t)} \\
& +\Gamma(P(t), U(t)),
\end{aligned}
$$

where $\Gamma(P(t), U(t))$ is defined in (73).

The parameters $a_{1}\left(x^{*}\right)$ and $a_{\mathrm{r}}\left(x^{*}\right)$ of the feedback control law (88) are the solutions of (23) and (24) for an assigned slope function value at the set point that satisfies (31). $P(t)=$ $x(t+D(\sigma(t), P(t)))$ is the prediction of the state at the time when the current control will have an effect on the state. Recall that the implicit integral relation (89) is derived from the inversion of the time variable $t \rightarrow t-D(t, x(t))$ in $t \rightarrow t+D(\sigma(t), P(t))$ with the prediction time defined as $\sigma(t)=t+D(\sigma(t), P(t))$. The key point of the design is the feasibility condition $\mathcal{F}_{c}$ defined in (56), which ensures that the control action can reach the plant, namely, the delay rate is bounded by unity. The a priori satisfaction of (56) depends on the magnitude $\epsilon$ and the angular frequency $\omega$ of the periodic instability, and on the design parameters of the extruder. 
Theorem 3

For any setpoint $x^{*} \in(0, L)$ and for any choosen setpoint slope $S\left(x^{*}\right) \in \mathbb{R}$ satisfying $S\left(x^{*}\right) \geq$ $S_{\min }\left(x^{*}\right)$, where $S_{\min }\left(x^{*}\right)$ is given by (31) and any initial condition $x_{0} \in[0, L)$ and,

$$
\left\{U_{0}(\theta) \mid U_{0}(\theta) \in\left[0, U_{m}\right], \text { for all } \theta \in\left[-D\left(t_{0}, x_{0}\right), 0\right)\right\},
$$

and any $U_{m}<1$, taking the control gains $a_{1}\left(x^{*}\right)$ and $a_{\mathrm{r}}\left(x^{*}\right)$ as solutions of (32) and (33), respectively, the closed-loop system consisting of the plant (71)-(73) with state $x(t)$, together with the control law (88)-(91), (22) with actuator state $U(t+\theta), \theta \in[-D(t, x(t)), 0)$, is GAS at $x=x^{*}$, $U=v\left(x^{*}\right)$ if the parameters of the extruder model and the perturbation satisfy,

$$
\begin{aligned}
0 & \leq \frac{\epsilon \omega}{(1-\epsilon)^{2}}<\frac{\theta_{1} \theta_{2}}{\left(1+\theta_{2} L\right)^{2}}, \\
\text { or, } & \frac{\theta_{1} \theta_{2}}{\left(1+\theta_{2} L\right)^{2}}<\frac{\epsilon \omega}{(1-\epsilon)^{2}}<\frac{\theta_{1}}{L}, \quad \text { and } \quad \theta_{2}<\frac{1}{L},
\end{aligned}
$$

or,

$$
\frac{\theta_{1} \theta_{2}}{\left(1+\theta_{2} L\right)^{2}}<\frac{\epsilon \omega}{(1-\epsilon)^{2}}<\frac{4 \theta_{1} \theta_{2}}{\left(1+\theta_{2} L\right)^{2}}, \quad \text { and } \quad \theta_{2}>\frac{1}{L}
$$

where, $\theta_{1}$ and $\theta_{2}$ are defined in (14) and (15), respectively.

Proof: The proof of Theorem 2 is based on the Lyapunov-like condition (56) that must be satisfied a priori to guarantee the GAS property for any given $x^{*} \in(0, L)$. In the following, we compute the function (49) for the time- and state-dependent input delay model of the extruder (71)-(73), (16), (17) in order to establish that the feasibility region as it is defined by (56) is the entire physical domain, namely, $x \in(0, L)$ and $U \in[0,1)$. First, (56) is satisfied if

$$
\frac{\partial D}{\partial t}(t, x)+\frac{\partial D}{\partial x}(t, x) f(t, x, U)<1,
$$

Using (87) and (86) together with (71)-(73), we rewrite (96) as

$$
\frac{\theta_{1} \epsilon \omega \sin (\omega t)}{c^{2}(t)}(L-x)+\Gamma(x, U)<1,
$$

The gradient of (73) with respect to the input $U$ satisfies

$$
\nabla_{U} \Gamma(x, U)=-\frac{\theta_{2}}{\left(1+\theta_{2} x\right)(1-U)^{2}},
$$

and indeed (73) is a strictly decreasing function of $U[0,1)$, for all $x(t) \in[0, \infty)$ and

$$
\sup _{U \in[0,1)} \Gamma(x, U)=\frac{\theta_{2} x}{\left(1+\theta_{2} x\right)} .
$$

Next, with the help of (99) and (87), we deduce that condition (56) is satisfied if,

$$
\Lambda(x)<1 \quad \forall x \in[0, L]
$$

where

$$
\Lambda(x)=\frac{\epsilon \omega(L-x)}{\theta_{1}(1-\epsilon)^{2}}+\frac{\theta_{2} x}{\left(1+\theta_{2} x\right)},
$$

Taking the derivative of (101) we get

$$
\Lambda^{\prime}(x)=\frac{\theta_{2}}{\left(1+\theta_{2} x\right)^{2}}-\frac{\epsilon \omega}{\theta_{1}(1-\epsilon)^{2}} .
$$

Since $\Lambda^{\prime}$ is a decreasing function with respect to $x$, for all $x \in[0, L]$, from (102), three different cases are possible for the sign of $\Lambda^{\prime}$, and thus also for the monotonicity of $\Lambda$. 
- Case 1: $\Lambda^{\prime}(x)>0 \forall x \in[0, L]$, i.e, $\Lambda(x)$ is strictly increasing with respect to $x$. Thus (100) is satisfied when

$$
\Lambda(L)=\frac{\theta_{2} L}{\left(1+\theta_{2} L\right)}<1
$$

The function $\Lambda^{\prime}(x)$ is strictly positive for all $[0, L]$ when

$$
\frac{\epsilon \omega}{(1-\epsilon)^{2}}<\frac{\theta_{1} \theta_{2}}{\left(1+\theta_{2} L\right)^{2}}
$$

$=$ Since $\omega>0$ and $0<\epsilon<1$, we obtain from (103) and (104) condition (93) of Theorem 3.

- Case 2: $\Lambda^{\prime}(x)<0, \forall x \in[0, L]$, i.e., $\Lambda(x)$ is strictly decreasing with respect to $x$. Thus

$$
\sup _{x \in[0, L]} \Lambda(x)=\frac{\epsilon \omega L}{\theta_{1}(1-\epsilon)^{2}} .
$$

The function $\Lambda^{\prime}(x)$ is strictly negative for all $x \in[0, L]$ when

$$
\frac{\epsilon \omega}{(1-\epsilon)^{2}}>\theta_{1} \theta_{2}
$$

Hence, by combining (105) and (106), (100) is satisfied when

$$
\theta_{1} \theta_{2}<\frac{\epsilon \omega}{(1-\epsilon)^{2}}<\frac{\theta_{1}}{L}
$$

One should notice that (107) necessarily restricts $\theta_{2}$ as

$$
\theta_{2}<\frac{1}{L}
$$

- Case 3: $\Lambda^{\prime}(x)>0, \forall x \in\left[0, x_{1}\right]$ and $\Lambda^{\prime}(x)<0, \forall x \in\left[x_{1}, L\right]$, thus $\Lambda^{\prime}\left(x_{1}\right)=0$ and $\Lambda(x)$ admits a maximum at $x=x_{1}$ (note that if $\Lambda^{\prime}(0)<0$, then $\Lambda^{\prime}(x)<0 \forall x \in[0, L]$ ). One gets

$$
x_{1}=(1-\epsilon) \sqrt{\frac{\theta_{1}}{\theta_{2} \epsilon \omega}}-\frac{1}{\theta_{2}} .
$$

Solution (109) is admissible if and only if $x_{1} \in(0, L)$, that is

$$
0<(1-\epsilon) \sqrt{\frac{\theta_{1}}{\theta_{2} \epsilon \omega}}-\frac{1}{\theta_{2}}<L,
$$

or, equivalently, if

$$
\frac{\theta_{1} \theta_{2}}{\left(1+\theta_{2} L\right)^{2}}<\frac{\epsilon \omega}{(1-\epsilon)^{2}}<\theta_{1} \theta_{2} .
$$

Moreover, using (109) one can conclude that (100) holds if

$$
\frac{\epsilon \omega}{(1-\epsilon)^{2}}<\frac{4 \theta_{1} \theta_{2}}{\left(1+\theta_{2} L\right)^{2}} .
$$

For satisfying (111) and (112) simultaneously, we need to either impose (95) or condition (111) together with condition $\theta_{2}<\frac{1}{L}$, which can be combined with conditions (107) and (108), into one condition given by (94). 


\section{Remark 1}

For given values for the parameters of the extruder, namely, $\theta_{1}, \theta_{2}$, and $L$, the condition (93) is always satisfied if $\epsilon$ or $\omega$ are sufficiently small. An increase of the magnitude of $\epsilon$ causes a decrease of the allowed $\omega$ and vice versa, as it is evident from (93)-(95). For given $\theta_{1}$, and $L$, the maximum bound of the perturbation parameters, namely, $\epsilon$ or $\omega$ is expressed in (93) as

$$
\sup _{\theta_{2} \in \mathbb{R}}\left\{\frac{\theta_{1} \theta_{2}}{\left(1+\theta_{2} L\right)^{2}}\right\}=\frac{\theta_{1}}{4 L}, \quad \theta_{2}=\frac{1}{L}
$$

Larger variations of $\epsilon$ and $\omega$ are possible, especially in the case in which $\theta_{2}$ is small, as it is evident from (94) and (95). However, for very large $\theta_{2}$, one can conclude from (93) and (95) that the allowable size of $\epsilon$ and $\omega$ is restricted. Moreover, from (93)-(95) one can conclude that the size of the allowable fluctuations of the transport speed in $\epsilon$ and $\omega$ is proportional to $\theta_{1}$ and inversely proportional to the extruder length $L$.

In physical terms, conditions (93)-(95) are mainly a correlation between the pressure and the "rotation" flow, namely, $\theta_{2}$ defined in (15) and $\theta_{1}$ defined in (14), respectively. We recall the expression of the net flow rate defined in (8) which is an increasing function of $\theta_{2}$ as it is shown in Figure 5. Therefore, changes in $\theta_{2}$, by manipulating $K_{d}, B$, or $\rho_{0}$, the nozzle conductance, the screw resistance, and the melt density, respectively, affect the output flow rate $F_{d}$. For example, an increase in $\theta_{2}$ by increasing the nozzle conductance $K_{d}$, leads to an increase in the outflow rate. Note that $K_{d}$, which defines the nozzle opening, is directly related to the printing resolution, namely, the accuracy of the printing process. A large nozzle opening leads to an extrusion of a filament with a large diameter and consequently deteriorates the printer precision. Moreover, from (94) and (15), it can be also seen that the "robustness" of the controller depends on the material thickness, namely, the mass density $\rho_{0}$ : a thicker material is less sensitive to large fluctuations of the transport speed under the predictor feedback control law. The parameter $B$ in the expression of $\theta_{2}$ in (15) is given by

$$
B=\frac{W H^{3}}{12},
$$

where $H$ is the approximate depth of screw channel from the screw thread root to the barrel internal surface, and $W$ is the width of screw channel. Consequently, changes in $\theta_{2}$ due to the changes in $B$ affect also the parameter $\theta_{1}$, since the screw pitch value $\xi$ also depends directly on $W$.

Relations (93)-(95) show that an increase in $\theta_{1}$, namely, an increase of the material convection speed, by enabling a large screw pitch $\xi$ or a high screw speed $N_{0}$, improves the "robustness" of the controller in some way and allows for a system that supports broader changes of the convection velocity in frequency and amplitude. Note that a sharp increase in the rotational screw speed $N_{0}$ results in material overload and clogging problems and has a major effect on the residence time that is the critical time during which the material should be heated to have good properties before being evicted through the nozzle. Particulary, the extruded filament homogeineity is directly related to the residence time and to the process of solidification after layers deposition in 3D printers. In addition, an increase in $\theta_{1}$ in the screw speed $N_{0}$, increases the thermal energy in the extruder chamber due to the material shearing and decreases the viscosity of the melt. In that case, a rapid feeding of the extruder with granular material by applying a more agressive "Bang-Bang" control action absorbs the excess heat in the system. Maintaining a reasonnable temperature inside the barrel is essential because an excessive overheating of the system burns the polymer or produces poor extrusion. Generally, the conventional extrusion processes are equipped with a cooling system to compensate for the heat generated by the mechanical shearing effect that is proportional to the screw speed.

In general, the nozzle and the screw designs are directly related to the predictor feedback control design and for achieving high performances for the closed-loop system the scale of the extruder should be neatly chosen. For instance, the agressiveness of the controller is influenced by the choice of $\theta_{2}$ since the minimum value of the slope at the set point $S_{\min }\left(x^{*}\right)$ defined in (31) 
depends on this parameter. Moreover, the entire process operates with an extruder head that moves very fast to print filament lines layer upon layer on a moving platform. A sufficiently light extruder head with small nozzle opening $K_{d}$ and a small length $L$ that operates at a sufficiently high screw speed $N_{0}$ is needed to ensure a high rate of extrusion with a high precision.

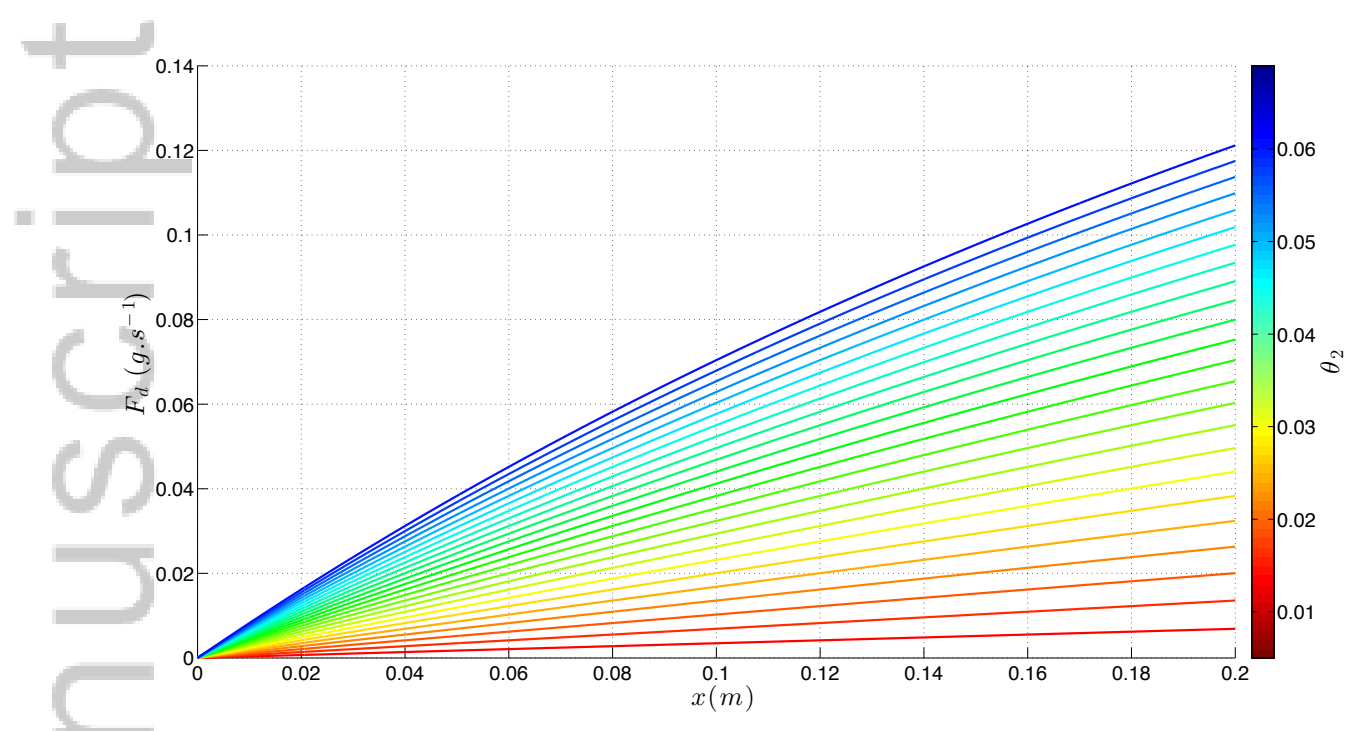

Figure 5. The nozzle flow rate is an increasing function of $\theta_{2}$.

\section{SIMULATIONS}

\subsection{Time- and state-dependent input delay compensation}

The setpoint is chosen as $x^{*}=0.16 \mathrm{~m}$ which corresponds to a desired nozzle output flow rate as indicated by the equation (8). The initial position of the moving interface is set to $x_{0}=0.1 \mathrm{~m}$, the total length of the extruder is $L=0.2 \mathrm{~m}$ and the system is supposed to settle at $x_{0}=0.1 \mathrm{~m}$ at the initial time. The value of the slope function is set to $S\left(x^{*}\right)=S_{\min }\left(x^{*}\right)+30$. The simulations show the dynamics of the input filling ratio $U(t)$, the interface position $x(t)$, the predictor state $P(t)$ and the delay function $D(t, x(t))$. Different cases including the open-loop dynamics, both uncompensated and compensated delay control laws are simulated for $\{\epsilon=0.2, \omega=4 \mathrm{rad} / \mathrm{s}\}$ and $\{\epsilon=0.65, \omega=1.2 \mathrm{rad} / \mathrm{s}\}$. It is clear that the uncompensated input leads to oscillatory responses and the compensated closed-loop control allows faster convergence than the open loop control. Also, as it is shown in Figure 10, the feasibility condition is satisfied in both presented simulation results.

\section{Remark 2}

We emphasize that the real time estimation of the delay can be achieved using a pressure sensor at the nozzle, (5) and (8) and with the knowledge of the variabilities parameters $\epsilon$ and $\omega$. In fact, in the recent papers [59, 60], the implementation issue of predictor feedback is discussed in detail and various numerical schemes are developed for computation of predictor feedback laws. In applications where the delay is not given explicitly as a function of measured quantities, such as, for example, in spark-ignited engines [61], numerical schemes for the real-time computation of the delay should be developed [61]. 

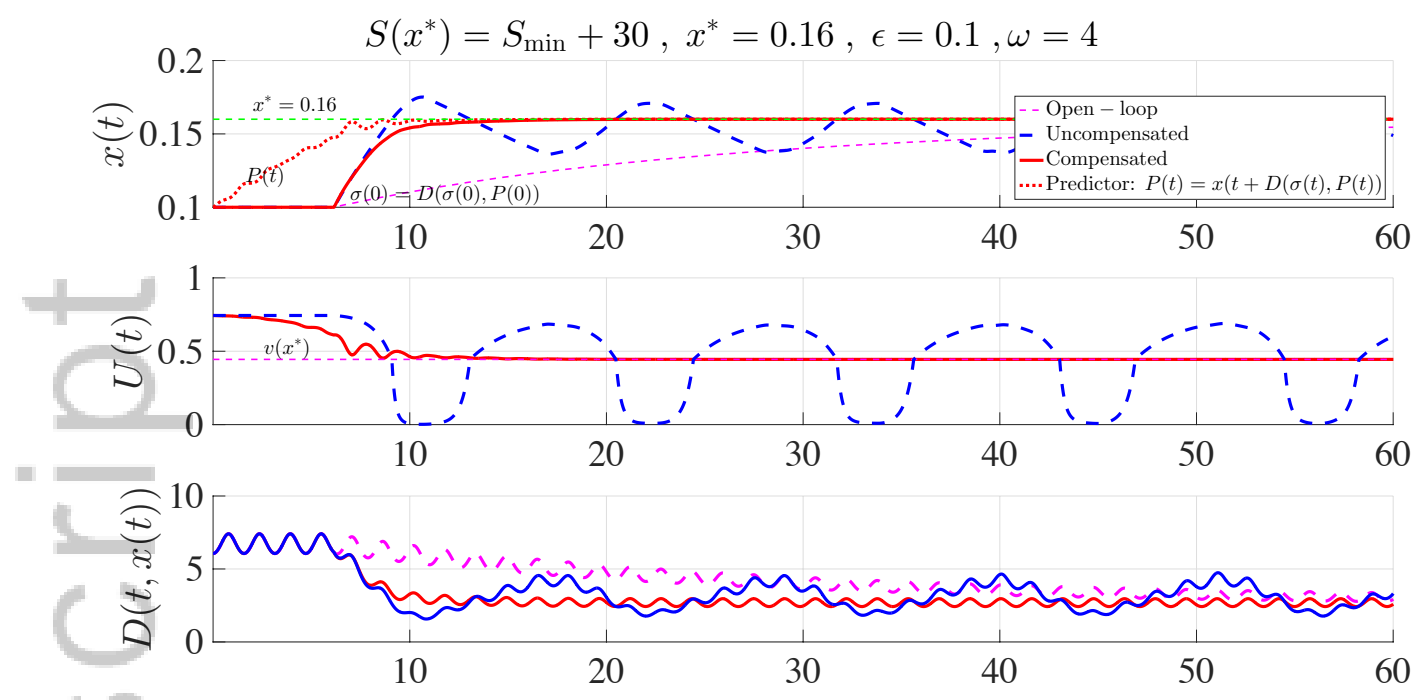

Figure 6. Compensation of the time- and state-dependent input delay-(a).
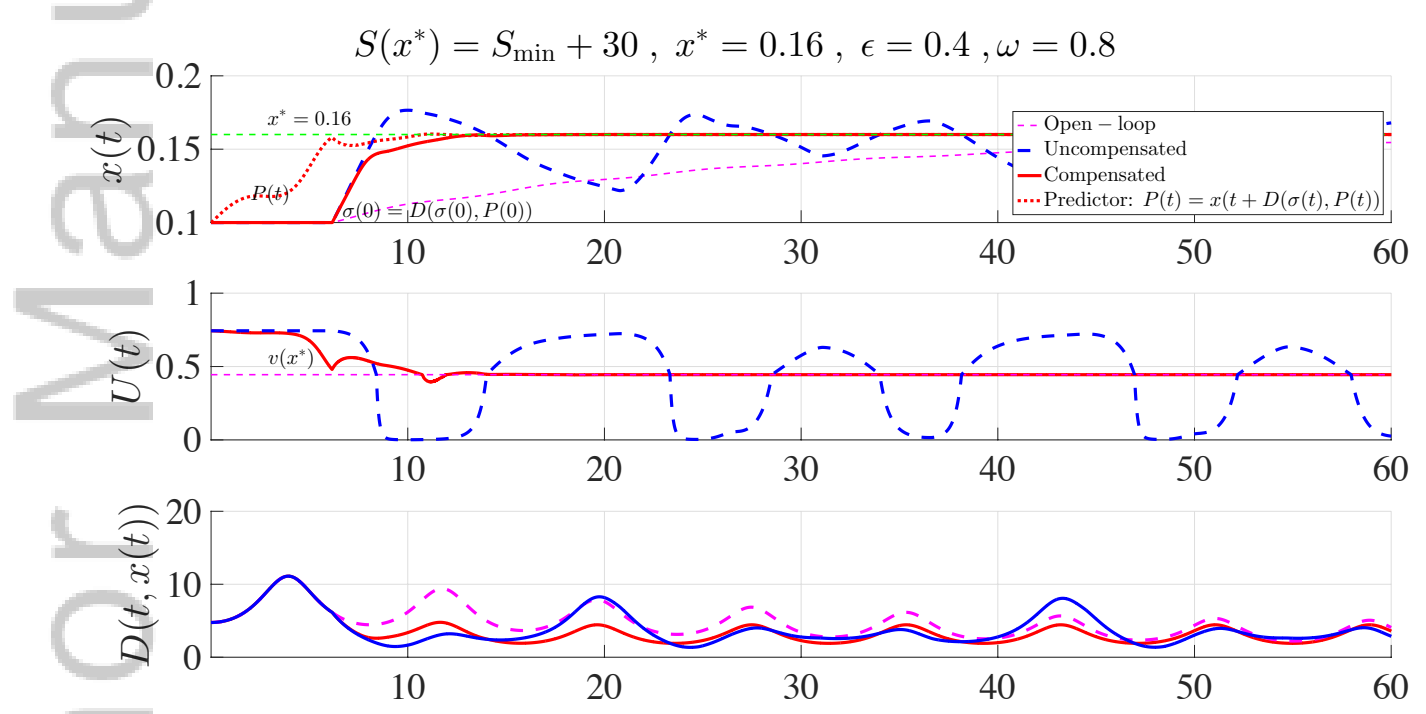

Figure 7. Compensation of the time- and state-dependent input delay-(b).

\subsection{The state-dependent input delay compensator for the model with constant viscosity}

The following simulation results show the stabilization of the model described by (11) with the state-dependent input delay predictor feedback law [35]. Defining (11) as

$$
\begin{aligned}
\dot{x}(t) & =f\left(x, U\left(t-D_{\mathrm{s}}(x(t))\right),\right. \\
D_{\mathrm{s}}(x(t)) & =\frac{L-x(t)}{\theta_{1}}
\end{aligned}
$$

where

$$
f(x(t), U(t))=-\theta_{1} \Gamma(x(t), U(t)),
$$


$\theta_{1}$ is the nominal transport velocity of the material defined in (14), and the function $\Gamma(x(t), U(t))$ is given by (73). The predictor feedback controller is written as

$$
\begin{aligned}
& U(t)=v\left(P_{\mathrm{s}}(t)\right), \\
& P_{\mathrm{s}}(t)=x(t)+\int_{t-D_{\mathrm{s}}(x(t))}^{t} \frac{f\left(P_{\mathrm{s}}(\mu), U(\mu)\right)}{1-F_{s}\left(P_{\mathrm{s}}(\mu), U(\mu)\right)} d \mu .
\end{aligned}
$$

where for all $t-D_{\mathrm{s}}(x(t)) \leq \mu \leq t$

$$
F_{s}\left(P_{\mathrm{s}}(\mu), U(\mu)\right)=\frac{\partial D_{\mathrm{s}}}{\partial x}\left(P_{\mathrm{s}}(\mu)\right) f\left(P_{\mathrm{s}}(\mu), U(\mu)\right) .
$$

By specializing Theorem 3 to the case $\epsilon=0$ it can be shown that the predictor feedback law (118)(120) renders system (115)-(117) GAS (in the physical domain) at any given equilibrium $x^{*}$.

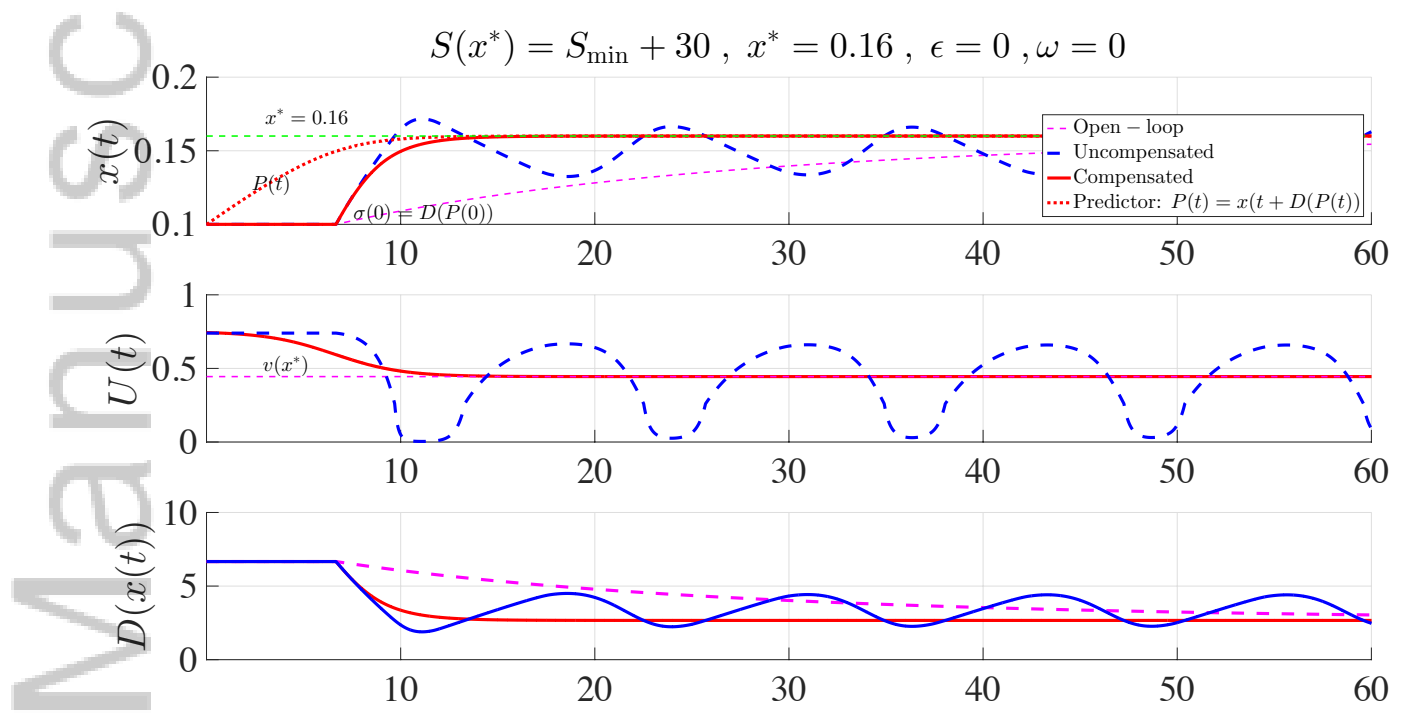

Figure 8. Compensation of the state-dependent input delay.

\subsection{Control with a state-dependent input delay compensator}

We deal with the case in which the time variations of the transport speed are unknown and consider the closed-loop system consisting of the plant (13) with an actual delay $D(t, x(t))$, given in (16), (17), together with a state- dependent input delay predictor feedback defined by

$$
\begin{aligned}
& U(t)=v(\hat{P}(t)), \\
& \hat{P}(t)=x(t)+\int_{t-\hat{D}(x(t))}^{t} \frac{\hat{f}(\hat{P}(s), U(s))}{1-\hat{F}(\hat{P}(s), U(s))} d s,
\end{aligned}
$$

where,

$$
\hat{F}(\hat{P}(s), U(s))=\frac{\partial \hat{D}}{\partial x}(\hat{P}(s)) \hat{f}(\hat{P}(s), U(s)) .
$$

With an estimated delay function defined as

$$
\hat{D}(x(t))=\frac{L-x(t)}{\xi N_{0}},
$$


and the nominal vector field,

$$
\hat{f}(x(t), U(t))=\theta_{1}\left[-\frac{\theta_{2} x(t)}{\left(1+\theta_{2} x(t)\right)(1-U(t))}+\frac{U(t)}{1-U(t)}\right], \quad U(t) \in[0,1) .
$$

More precisely, both the predictor state, $\hat{P}(t)$ and the delay function, $\hat{D}(x(t))$, are estimates of the actual prediction state, namely, $P(t)$, and delay function, namely, $D(t, x(t))$, that are described by (89) and (16), (17), respectively. For implementing the controller (121), (122), the "actual" feasibility condition, defined in (93), (94), and (95) have to hold, in order to guarantee that the controller actually "kicks in". In addition, we assume that the following condition, which guarantees that the denominator in (122) remains always positive (and hence, the controller remains bounded) is satisfied

$$
\frac{\theta_{2} \hat{P}(\theta)}{\left(1+\theta_{2} \hat{P}(\theta)\right)(1-U(\theta))}-\frac{U(\theta)}{(1-U(\theta))}<1, \quad \theta \in[t-\hat{D}(x(t)), t] .
$$

Note that with strictly positive physical parameters $B, \rho_{0}$, and $K_{d}$, and for $U \in\left[0, v_{\max }\right]$, relation (126) is satisfied whenever $\hat{P}(\theta) \in[0, \infty)$, for all $\theta \in[t-\hat{D}(x(t)), t]$.

The simulation results in Figure 9 illustrate that the state-dependent input delay compensator can handle small time-varying uncertainties on the vector field (125) and the delay function (124), as described by (72)-(73) and (16)-(17), respectively. An increase in the necessary control effort to drive the system to the setpoint is also denoted and the rate of convergence decreases compared to the time- and state-dependent predictor feedback (88)-(90) shown in Figure 7.

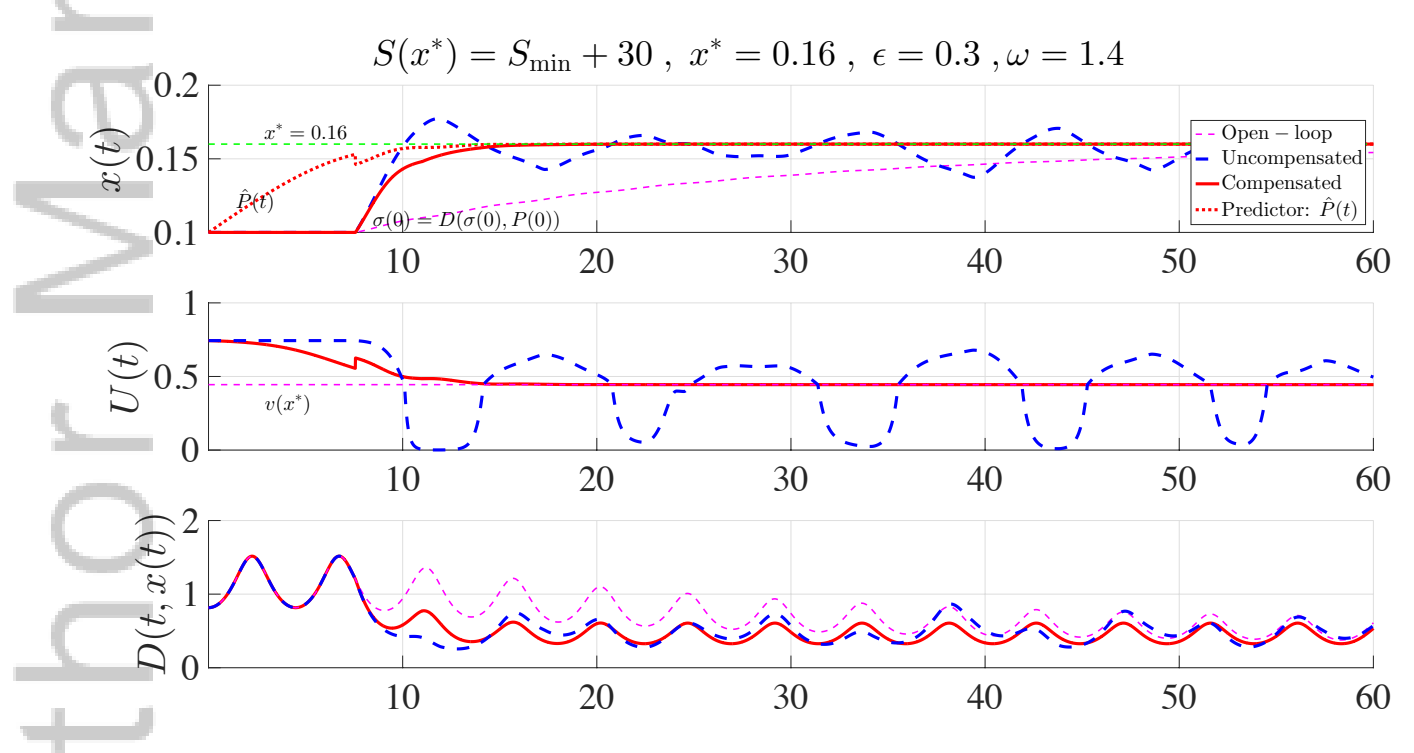

Figure 9. Robustness of the state-dependent input delay compensator.

\section{CONCLUSIONS}

This paper is devoted to the stabilization of a screw extrusion process. A coupled PDE-ODE model is used to derive a state-dependent input delay system describing the melt convection in the extruder chamber for an isothermal case. The extension of the aforementioned model to a nonisothermal case is proposed introducing a periodic time-dependent function in the state-dependent input delay function. Next, we design a predictor feedback controller to compensate the state- and 


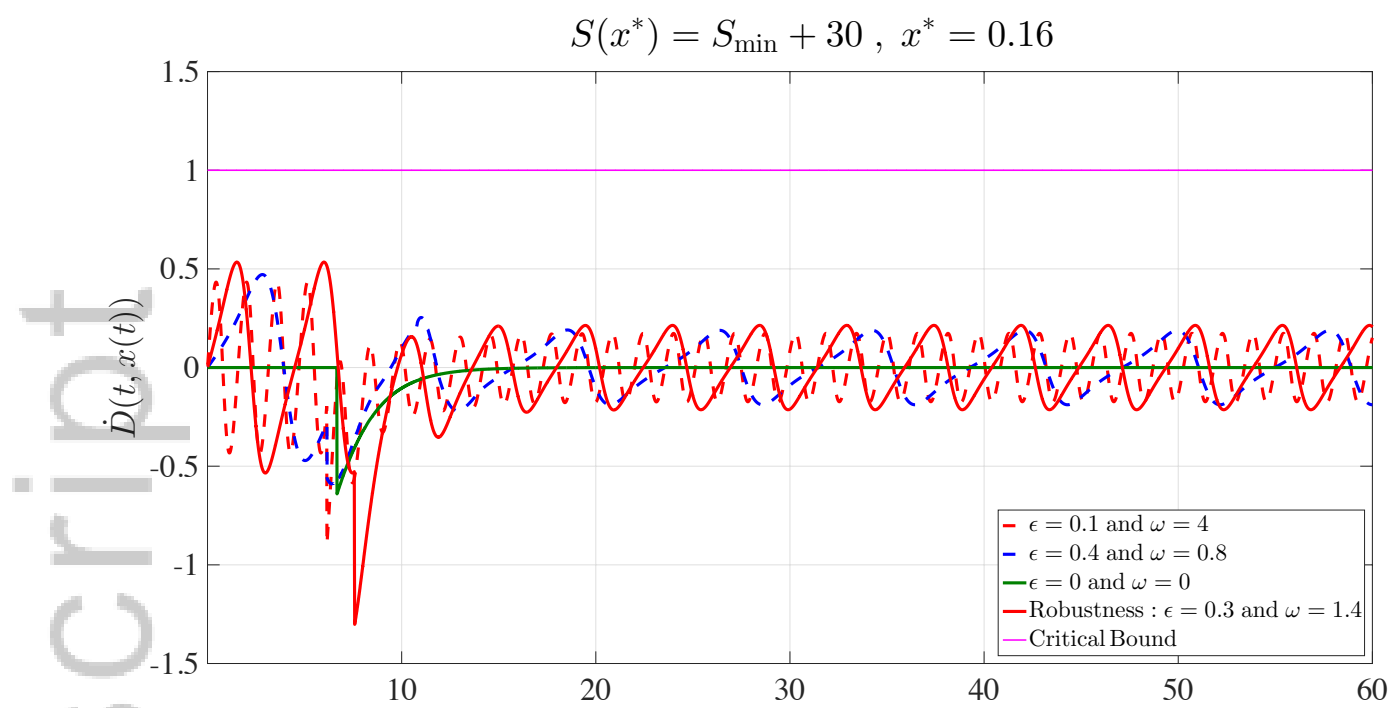

Figure 10. Feasibility condition: the delay rate is bounded by unity.

time-dependent input delay and establish the GAS of any setpoint with respect to the physical domain under physical and design restrictions. The delay compensator is constructed with a nominal Bang-Bang-like controller that ensures the GES of the delay-free plant.

It is clear that the delay function model for the non-isothermal extrusion process should depend on the rheological properties of the extruded polymer. In general, a fairly accurate estimation of the material friction and the viscosity behavior is extremely hard to achieve in such processes due to the change in material composition, and the strong interaction between the heat and mass transfer phenomena. An interesting future work would be to consider an unknown time-dependent perturbation acting on the polymer convection speed. As it is shown in the simulation results, the state-dependent compensator might be able to handle time-varying perturbations acting on the vector field and the delay function. The proof of the robustness properties of the state-dependent input delay predictor is an interesting perspective.

\section{Physical definition of the parameters}

$\begin{array}{lll}L=200.10^{-3} & m & \text { Extruder length } \\ N_{0}=90 & \mathrm{rpm} & \text { Screw speed } \\ B=9.345010^{-9} & \mathrm{~m}^{4} & \text { Geometric parameter } \\ F_{d}=-- & \mathrm{Kg} \mathrm{s} \mathrm{s}^{-1} & \text { Net forward mass flow rate } \\ K_{d}=2.4510^{-5} & \mathrm{~kg} & \text { Nozzle conductance } \\ \xi=1010^{-3} & \mathrm{~m} & \text { Screw Pitch } \\ S_{\text {eff }}=-- & \mathrm{m}^{2} & \text { Effective area } \\ V_{\text {eff }}=\xi S_{\text {eff }} & \mathrm{m}^{3} & \text { Effective volume } \\ \eta=-- & \mathrm{Pa} \mathrm{s}^{-1} & \text { Melt viscosity } \\ \rho_{0}=1240 & \mathrm{Kg} \mathrm{m}^{-3} & \text { PLA Melt density } \\ \epsilon=-- & -- & \text { Amplitude of the perturbation } \\ \omega=-- & \mathrm{rad.s} \mathrm{s}^{-1} & \text { Frequency }\end{array}$

APPENDIX

Below are presented Lemmas 1-8 for the proof of Theorem 2. 
Lemma 1 (Backstepping Transformation of the Actuator State)

The infinite dimensional backstepping transform of the actuator state given by

$$
W(\theta)=U(\theta)-\kappa(\sigma(\theta), P(\theta)),
$$

for all $\phi(t) \leq \theta \leq t$, allows to transform the system (43) with the controller (47)-(46) into the following target system

$$
\begin{aligned}
& \dot{x}(t)=f(t, x(t), \kappa(t, x(t))+W(\phi(t)) \\
& W(t)=0,
\end{aligned}
$$

where $\phi(t)$ is defined in (44).

Proof: The proof of Lemma 1 is based on a direct verification considering $P(\phi(t))=x(t)$ and $\sigma(\phi(t))=t$ in the original system (43).

Lemma 2 (Inverse Backstepping Transformation)

The inverse of the infinite dimensional backstepping transormation (A-1) is defined for all $\phi(t) \leq$ $\theta \leq t$ by

$$
U(\theta)=W(\theta)+\kappa(\bar{\sigma}(\theta), \Pi(\theta))
$$

with

$$
\begin{aligned}
\Pi(\theta) & =x(t)+\int_{\phi(t)}^{\theta} \frac{f(\bar{\sigma}(s), \Pi(s), \kappa(\bar{\sigma}(s), \Pi(s))+W(s))}{1-F(\bar{\sigma}(s), \Pi(s), W(s))} d s \\
F(\bar{\sigma}(s), \Pi(s), W(s)) & =\frac{\partial D}{\partial t}(\bar{\sigma}(s), \Pi(s))+\frac{\partial D}{\partial x}(\bar{\sigma}(s), \Pi(s)) f(\bar{\sigma}(s), \Pi(s), \kappa(\bar{\sigma}(s), \Pi(s))+W(s)) \\
\bar{\sigma}(\theta) & =t+\int_{\phi(t)}^{\theta} \frac{1}{1-F(\bar{\sigma}(s), \Pi(s), W(s))} d s,
\end{aligned}
$$

where $\phi(t)$ is defined in (44).

Proof: Direct verification considering that $P(\theta)=\Pi(\theta)$ and $\bar{\sigma}(\theta)=\sigma(\theta)$ for all $\phi(t) \leq \theta \leq t$. We refer to $P(\theta)$ as the plant-predictor system and $\Pi(\theta)$ as the target-predictor system, respectively. However, they play different roles because they are driven by different inputs ( $U$ versus $W$ ).

Lemma 3 (Stability of the Target System)

There exists a class $\mathcal{K} \mathcal{L}$ function $\beta$ such that for all solutions of the system satisfying the feasibility condition (56), the following holds:

$$
\begin{aligned}
& \Xi(t) \leq \beta\left(\Xi\left(t_{0}\right), t-t_{0}\right), \quad t \geq t_{0} \\
& \Xi(t)=|x(t)|+\sup _{\phi(t) \leq \theta \leq t}|W(\theta)|,
\end{aligned}
$$

where $\phi(t)$ is defined in (44).

Proof: Under Assumption 2, from [2] and [62] (see also [1, 3]), there exist a continuously differentiable function $S: \mathbb{R}_{+} \times \mathbb{R}^{n} \rightarrow \mathbb{R}_{+}$and class $\mathcal{K}_{\infty}$ functions $\alpha_{1}, \alpha_{2}, \alpha_{3}, \alpha_{4}$ such that for any $g>0$

$$
\begin{aligned}
& \alpha_{1}(|x(t)|) \leq S(t, x(t)) \leq \alpha_{2}(|x(t)|), \\
& \dot{S}(t, x(t)) \leq-g S(t, x(t))+\alpha_{3}(|W(\phi(t))|),
\end{aligned}
$$

with

$$
\dot{S}(t, x(t))=\frac{\partial S(t, x(t))}{\partial t}+\frac{\partial S(t, x(t))}{\partial x} f(t, x(t), \kappa(t, x(t))+W(\phi(t))) .
$$


Let us define a functional as

$$
L_{g, n}(t)=\int_{\phi(t)}^{t} e^{2 n g(\sigma(\theta)-t)} W(\theta)^{2 n} d \theta
$$

for any positive integer $n$ and any $g>0$. The time derivative of (A-13) along (A-2), (A-3) is written as

$$
\dot{L}_{g, n}(t)=-\frac{d \phi(t)}{d t} e^{2 n g(\sigma(\phi(t))-t)} W(\phi(t))^{2 n}-2 n g \int_{\phi(t)}^{t} e^{2 n g(\sigma(\theta)-t)} W(\theta)^{2 n} d \theta .
$$

From (56) it follows that $\dot{\phi}(t)>0$, for all $t \geq t_{0}$, and thus the following inequality holds for all $t \geq t_{0}$

$$
\dot{L}_{g, n}(t) \leq-2 n g L_{g, n}(t) .
$$

Let us now define for any $g>0$ the functional

$$
L(t)=\int_{\phi(t)}^{t} e^{g(\sigma(\theta)-t)} \bar{\gamma}(|W(\theta)|) \frac{1}{\phi^{\prime}(\sigma(\theta))} d \theta,
$$

for any class $\mathcal{K}_{\infty}$ function $\bar{\gamma}$. Knowing that $\sigma(\phi(t))=t$, the derivative of $L$ with respect to time along (A-2), (A-3) is written as

$$
\dot{L}(t)=-\bar{\gamma}(\mid W(\phi(t) \mid)-g L(t)
$$

Next, defining the functional

$$
V_{1}(t)=S(t, x(t))+L(t)
$$

whose time derivative along (A-2) is written as

$$
\dot{V}_{1}(t)=\frac{\partial S(t, x(t))}{\partial t}+\frac{\partial S(t, x(t))}{\partial x} f(x(t), \kappa(x(t))+W(\phi(t)))+\dot{L}(t),
$$

and combining (A-11) with (A-17), we obtain the following inequality

$$
\dot{V}_{1}(t) \leq-g(S(t, x(t))+L(t))+\alpha_{3}(|W(\phi(t))|)-\bar{\gamma}(|W(\phi(t))|) .
$$

Choosing $\bar{\gamma}$ such that $\bar{\gamma}(s) \geq \alpha_{3}(s)$, for all $s \geq 0$, we get

$$
\dot{V}_{1}(t) \leq-g V_{1}(t)
$$

Let us define next the Lyapunov functional for the target system (A-2) and (A-3) as

$$
V_{g, n}(t)=V_{1}(t)^{2 n}+L_{g, n}(t)
$$

Taking the derivative of $V_{g, n}$ with the help of (A-15) and (A-21) we get

$$
\dot{V}_{g, n}(t) \leq-2 n g V_{g, n}(t)
$$

Therefore,

$$
V_{g, n}(t)^{\frac{1}{2 n}} \leq e^{-g\left(t-t_{0}\right)} V_{g, n}\left(t_{0}\right)^{\frac{1}{2 n}}
$$

It then follows that

$$
V_{1}(t)+L_{g, n}(t)^{\frac{1}{2 n}} \leq 2 e^{-g\left(t-t_{0}\right)}\left(V_{1}\left(t_{0}\right)+L_{c, n}\left(t_{0}\right)^{\frac{1}{2 n}}\right) .
$$


From (A-13), the following holds

$$
L_{g, n}(t)^{\frac{1}{2 n}}=\left(\int_{\phi(t)}^{t} e^{2 n g(\sigma(\theta)-t)} W(\theta)^{2 n} d \theta\right)^{\frac{1}{2 n}},
$$

Thus, taking the limit as $n \rightarrow \infty$ of (A-26) and using the fact that

$$
\lim _{n \rightarrow \infty} L_{g, n}(t)^{\frac{1}{2 n}}=\sup _{\phi(t) \leq \theta \leq t}\left|e^{g(\sigma(\theta)-t)} W(\theta)\right| \equiv\|W(t)\|_{g, \infty},
$$

we conclude that the following holds

$=V_{1}(t)+\|W(t)\|_{g, \infty} \leq 2 e^{-g\left(t-t_{0}\right)}\left(V_{1}\left(t_{0}\right)+\left\|W\left(t_{0}\right)\right\|_{g, \infty}\right)$.

From (A-16) and noting that $\phi^{\prime}(t)>0$, for all $t \geq t_{0}$, it follows that

$$
L(t) \leq \sup _{\phi(t) \leq \theta \leq t}\left|e^{g(\sigma(\theta)-t)} \bar{\gamma}(|W(\theta)|)\right| \int_{\phi(t)}^{t} \frac{1}{\phi^{\prime}(\sigma(\theta))} d \theta .
$$

Noting that $\dot{\sigma}(\theta)=\frac{1}{\phi^{\prime}(\sigma(\theta))}$, the following holds

$$
L(t) \leq(\sigma(t)-t) \sup _{\phi(t) \leq \theta \leq t}\left|e^{g(\sigma(\theta)-t)} \bar{\gamma}(|W(\theta)|)\right| .
$$

Using the feasibility condition (56) and (55) we deduce

$$
\dot{\sigma}(\theta) \leq \frac{1}{1-c} .
$$

By integration of (A-31) on $[\phi(t), t]$ and using the fact that $\sigma(\phi(t))=t$, we derive the inequality

$$
\sigma(t)-t \leq \frac{1}{1-c} D(t, x(t)) .
$$

From Assumption 3 and (60) the following inequality holds

$$
\sigma(t)-t \leq \frac{1}{1-c}\left(c_{1}+\mu_{4}(|x(t)|)\right) .
$$

Hence, from (A-30) it follows that

$$
L(t) \leq \frac{1}{1-c}\left(c_{1}+\mu_{4}(|x(t)|)\right) e^{g\left(c_{1}+\mu_{4}(|x(t)|)\right)} \sup _{\phi(t) \leq \theta \leq t} \bar{\gamma}(|W(\theta)|),
$$

and thus,

$$
L(t) \leq \frac{1}{1-c}\left(c_{1}+\mu_{4}(|x(t)|)\right) e^{g\left(c_{1}+\mu_{4}(|x(t)|)\right)} \bar{\gamma}\left(\sup _{\phi(t) \leq \theta \leq t}|W(\theta)|\right),
$$

From the definition of $V_{1}$ in (A-18), using the facts that

$$
\sup _{\phi(t) \leq \theta \leq t}|W(\theta)| \equiv\|W(t)\|_{\infty} \leq\|W(t)\|_{g, \infty} \leq e^{g\left(c_{1}+\mu_{4}(|x|)\right)}\|W(t)\|_{\infty},
$$

and that $S(t, x(t)) \leq V_{1}(t)$, which follow from the fact that $\dot{\sigma}(\theta)>0$ for all $\theta \geq \phi\left(t_{0}\right)$, together with (A-10) and (A-34) we get from (A-28) that

$$
\begin{aligned}
\alpha_{1}(|X(t)|) & +\|W(t)\|_{\infty} \leq 2 e^{-g\left(t-t_{0}\right)}\left(\alpha_{2}\left(\left|x\left(t_{0}\right)\right|\right)\right. \\
& +\frac{1}{1-c}\left(c_{1}+\mu_{4}(|x(t)|)\right) e^{g\left(c_{1}+\mu_{4}(|x(t)|)\right)} \bar{\gamma}\left(\left\|W\left(t_{0}\right)\right\|_{\infty}\right) \\
& \left.+e^{g\left(c_{1}+\mu_{4}(|x|)\right)}\left\|W\left(t_{0}\right)\right\|_{\infty}\right)
\end{aligned}
$$

With the properties of comparison functions, we conclude that there exists a class $\mathcal{K} \mathcal{L}$ function $\beta$ such that (A-8) holds. 
Lemma 4 (Bound of the Predictor in Terms of Actuator State)

There exists a class $\mathcal{K}_{\infty}$ function $\rho$ such that for all the solutions of the system satisfying the feasibility condition (56), the following holds for all $\phi(t) \leq \theta \leq t$

$$
|P(\theta)| \leq \rho\left(|x(t)|+\sup _{\phi(t) \leq s \leq t}|U(s)|\right),
$$

where $\phi(t)$ is defined in (44).

Proof: Differentiating (47), we deduce the following relation for all $\phi(t) \leq \theta \leq t$

$$
\begin{aligned}
\frac{d P(\theta)}{d \theta} & =\frac{f(\sigma(\theta), P(\theta), U(\theta))}{1-F(\sigma(\theta), P(\theta), U(\theta))}, \\
F(\sigma(\theta), P(\theta), U(\theta)) & =\frac{\partial D}{\partial x} D(\sigma(\theta), P(\theta)) f(\sigma(\theta), P(\theta), U(\theta))+\frac{\partial D}{\partial t}(\sigma(\theta), P(\theta)),
\end{aligned}
$$

and with the change of variable $y=\sigma(\theta)$, (A-39) may be rewritten as:

$$
\frac{d P(\phi(y))}{d y}=f(y, P(\phi(y)), U(y-D(\phi(y)))), \quad t \leq y \leq \sigma(t) .
$$

From Assumption 1 and (56), which implies that $\dot{\sigma}(\theta)>0$, for all $\phi(t) \leq \theta \leq t$, we get that

$$
\frac{d R(y, P(\phi(y)))}{d \theta} \leq \dot{\sigma}(\theta)\left(\chi R(y, P(\phi(y)))+\mu_{3}(|U(y-D(\phi(y)))|)\right),
$$

for all $t \leq y \leq \sigma(t)$ and using the feasibility condition (56), we deduce, for all $\phi(t) \leq \theta \leq t$.

$$
\frac{d R(\sigma(\theta), P(\theta))}{d \theta} \leq \frac{1}{1-c}\left(\chi R(\sigma(\theta), P(\theta))+\mu_{3}(|U(\theta)|)\right) .
$$

By Assumption 3 and the comparison principle, we obtain

$$
R(\sigma(\theta), P(\theta)) \leq e^{\frac{\chi}{1-c}\left(c_{1}+\mu_{4}(|x|)\right)}\left(R(t, x(t))+\frac{1}{\chi} \sup _{\phi(t) \leq s \leq t} \mu_{3}(|U(s)|)\right), \phi(t) \leq \theta \leq t .
$$

With the standard properties of class $\mathcal{K}_{\infty}$ functions the Lemma (4) is deduced and the class $\mathcal{K}_{\infty}$ function $\rho$ is written as:

$$
\rho(s)=\mu_{1}^{-1}\left(\left(\mu_{2}(s)+\frac{1}{\chi} \mu_{3}(s)\right) e^{\frac{\chi}{1-c}\left(c_{1}+\mu_{4}(s)\right)}\right) .
$$

Lemma 5 (Bound of the Predictor in Terms of Transformed Actuator State)

There exists a class $\mathcal{K}$ function $\psi$ such that for all the solutions of the system satisfying the feasibility condition (56), the following holds:

$$
|\Pi(\theta)| \leq \psi\left(|x(t)|+\sup _{\phi(t) \leq s \leq t}|W(s)|\right), \quad \text { for all } \phi(\mathrm{t}) \leq \theta \leq \mathrm{t},
$$

where $\phi(t)$ is defined in (44).

Proof: The plant $\dot{x}(t)=f(t, x(t), \kappa(t, x(t))+\omega(t))$ satisfying the uniform input-to-state stability property with respect to $\omega$, and the function $\kappa$ being locally Lipschitz in both arguments and uniformly bounded with respect to its first argument, there exist a class $\mathcal{K} \mathcal{L}$ function $\beta_{2}$ and a class $\mathcal{K}$ function $\psi_{1}$ such that for all $\tau \geq t_{0}$

$$
Y(\tau) \leq \beta_{2}\left(\left|Y\left(t_{0}\right)\right|, \tau-t_{0}\right)+\psi_{1}\left(\sup _{s \geq t_{0}}|\omega(s)|\right),
$$


with

$$
\dot{Y}(\tau)=f(Y(\tau), \kappa(\tau, Y(\tau))+\omega(\tau)) .
$$

Now, we consider the change of variable $y=\sigma(\theta)$ and write the predictor of the target system (A-5) as

$$
\frac{d \Pi(\phi(y))}{d y}=f(y, \Pi(\phi(y)), \kappa(y, \Pi(\phi(y)))+\omega(\phi(y))), \quad t \leq y \leq \sigma(t) .
$$

Using (A-46), we derive the following relation

$$
=\sqrt{2}|\Pi(\theta)| \leq \psi_{2}(|x(t)|)+\psi_{1}\left(\sup _{\phi(t) \leq s \leq t}|W(s)|\right),
$$

for all $\phi(t) \leq \theta \leq t$ with a class $\mathcal{K}$ function $\psi_{2}(s)=\beta_{2}(s, 0)$. Using the properties of class $\mathcal{K}$ functions, (A-44) is deduced with $\psi(s)=\psi_{1}(s)+\psi_{2}(s)$.

Lemma 6 (Equivalence of the Norms of the Original and the target system)

There exist class $\mathcal{K}_{\infty}$ functions $\rho_{1}, \mu_{7}$ such that for all the solutions of the system satisfying the feasibility condition (56) and for all $t \geq t_{0}$, the following hold:

$$
\begin{aligned}
& \Omega(t) \leq \mu_{7}^{-1}(\Xi(t)), \\
& \Xi(t) \leq \rho_{1}(\Omega(t)),
\end{aligned}
$$

where $\Omega$ and $\Xi$ are defined in (64) and (A-9), respectively.

Proof: Using the inverse transformation (A-4) and the bound (A-44), we derive (A-49) with

$$
\mu_{7}^{-1}(s)=s+\hat{\rho}(\psi(s)),
$$

and from the direct transformation (A-1) together with the bound (A-38), we deduce (A-50), where $\rho_{1}$ is define as

$$
\rho_{1}(s)=s+\hat{\rho}(\rho(s))
$$

Lemma 7 (Ball Around the Origin Within the Feasibility Region)

There exists a positive constant $\bar{\gamma}$ such that for all the solutions of the system that satisfy

$$
|x(t)|+\sup _{\phi(t) \leq \theta \leq t}|U(\theta)|<\bar{\gamma},
$$

the feasibility condition (56) is satisfied.

Proof: From (45) we derive the following inequality

$$
|f(t, x(t), U(\phi(t)))| \leq \hat{\alpha}\left(|x(t)|+\sup _{\phi(t) \leq s \leq t}|U(s)|\right)
$$

Recalling the relations (61) and ( 62) of Assumption 3, we deduce that for all $\theta \in[\phi(t), t]$ and $c \in] 0,1[$, if a solution satisfies

$$
c_{3}+\mu_{5}(|P(\theta)|)+\left(c_{2}+\mu_{6}(|P(\theta)|)\right) \hat{\alpha}\left(|P(\theta)|+\sup _{\phi(t) \leq s \leq t}|U(s)|\right)<c,
$$

then it also satisfies (56).

Using Lemma 4 we conclude that (A-55) is satisfied if the following holds

$$
\left(c_{2}+\mu_{6}(\rho(\Omega(t)))\right) \hat{\alpha}(\rho(\Omega(t))+\Omega(t))+\mu_{5}(\rho(\Omega(t)))<c-c_{3} .
$$


Let us define a class $\mathcal{K}_{\infty}$ function $\rho_{c}$ as

$$
\rho_{c}(s)=\mu_{5}(\rho(s))+\left(c_{3}+\mu_{6}(\rho(s))\right) \hat{\alpha}(\rho(s)+s) .
$$

It follows that

$$
\bar{\gamma}=\rho_{c}^{-1}\left(c-c_{3}\right)
$$

Lemma 8 (Estimate of the Region of Attraction)

There exists a class $\mathcal{K}$ function $\psi_{\text {RoA }}$ such that for all initial conditions of the closed-loop system that satisfy relation (63), the solutions of the system satisfy (A-53) for $c \in] 0,1[$, and hence, satisfy (56).

Proof: Using Lemma 6 and (A-8), the following holds:

$$
\Omega(t) \leq \mu_{7}^{-1}\left(\beta\left(\rho_{\star}\left(\rho_{1}\left(\Omega\left(t_{0}\right)\right)\right), t-t_{0}\right)\right),
$$

where $\Omega$ is defined in (64). Introducing the class $\mathcal{K}_{\infty}$ function $\mu_{9}(s)=\mu_{7}^{-1}(\beta(s, 0))$, we derive the inequality

$$
\Omega(t) \leq \mu_{9}\left(\rho_{\star}\left(\rho_{1}\left(\Omega\left(t_{0}\right)\right)\right)\right) .
$$

Hence, for all initial conditions that satisfy the bound (56) with any class $\mathcal{K}$ choice

$$
\psi_{R o A}\left(c-c_{3}\right) \leq \rho_{1}^{-1}\left(\rho_{*}^{-1}\left(\mu_{9}^{-1}\left(\rho_{c}^{-1}\left(c-c_{3}\right)\right)\right)\right),
$$

the solutions satisfy (A-53). Moreover, for all of those initial conditions, the solutions verify (64), for all $\theta>\phi\left(t_{0}\right)$.

\section{REFERENCES}

1. Karafyllis I., and Jiang, Z.-P. Stability and Stabilization of Nonlinear Systems. Springer-Verlag London. 2011.

2. Malisoff, M., Mazenc, F. Further remarks on strict input-to-state stable Lyapunov functions for time-varying systems. Automatica 2005; 41(11):1973-1978.

3. Edwards, H., Lin, Y., Wang, Y. Further remarks on strict input-to-state stable Lyapunov functions for time-varying systems. in Proc. 39th IEEE Conf. Decision and Control, Sydney, Australia 2000; 3501-3506.

Edwards, H., Y. Lin, Y. Wang, On input-to-state stability for time varying nonlinear systems, in Proc. 39th IEEE Conf. Decision and Control, Sydney, Australia, 2000, pp. 3501-3506.

4. Mironov V., Boland T., Trusk T., Forgacs G., Markwald R. R. Organ printing: computer-aided jet-based 3d tissue engineering. Trends in Biotechnology 2003; 21(4):157-161.

5. Billiet T. B., Gevaert E., De Schryver T., Cornelissen M., Dubruel P. The 3D printing of gelatin methacrylamide cell-laden tissue-engineered constructs with high cell viability. Biomaterials 2014; 35(1):49-62.

6. Dragone V, Sans V, Rosnes M. H, Kitson P. J, Cronin L. 3d-printed devices for continuous-flow organic chemistry. Beilstein Journal of Organic Chemistry 2013; 9:951-959.

7. Valkenaers H, Vogeler F, Ferraris E, Voet A, Kruth J. P. A novel approach to additive manufacturing: screw extrusion

3d-printing. 10th International Conference on Multi Material Micro Manufacturing, 2013; 235-238.

8. Ladd C, So J. H., Muth J., Dickey M. D. 3D printing of free standing liquid metal microstructures. Advanced Materials 2013; 25(36).

9. Seitz H, Rieder W, Irsen S, Leukers B, Tille C. Three-dimensional printing of porous ceramic scaffolds for bone tissue engineering. Journal of Biomedical Materials Research Part B: Applied Biomaterials 2005; 74B(2):782-788.

10. Widmer M. S., Gupta P. K., Lu L., Meszlenyi R. K, Evans G. R, Brandt K, Savel T, Gurlek A, Patrick Jr C. W., Mikos A. G. Manufacture of porous biodegradable polymer conduits by an extrusion process for guided tissue regeneration. Biomaterials 1998; 19(21):1945-1955.

11. Zein I., Hutmacher D. W, Tan K. C. and Teoh S. H. Fused deposition modeling of novel scaffold architectures for tissue engineering applications. Biomaterials 2002; 23(4):1169 - 1185.

12. Hutmacher D. W., Schantz T., Zein I, Ng K. W., Teoh S. H, Tan K. C. Mechanical properties and cell cultural response of polycaprolactone scaffolds designed and fabricated via fused deposition modeling. Journal of Biomedical Materials Research 2001; 55(2):203-216.

13. Wei H, Xianglin Z, Quan W, Bin W. Fabrication of ha/ $\beta$-tcp scaffolds based on micro-syringe extrusion system. Rapid Prototyping Journal 2013; 19(5):319-326.

14. Deuser B. K, Tang L, Landers R. G., Leu M. C., Hilmas G. E. Hybrid extrusion force-velocity control using freeze-form extrusion fabrication for functionally graded material parts. Journal of Manufacturing Science and Engineering 2013; 35:1 - 11. 
15. Li M, Tang L, Xue F, Landers R. G. Numerical simulation of ram extrusion process for ceramic materials. Proceedings of Solid Freeform Symposium, Austin, TX, vol. 35, 2011; 290-308.

16. Zhao X, Landers R. G., Leu M. C. Adaptive control of freeze-form extrusion fabrication processes. Proceedings of ASME Dynamic Systems and Control Conference, Ann Arbor, vol. 35, 2008; 290-308.

17. Mason M. S., Huang T., Landers R. G., Leu M.C., Hilmas G. E., Hayes M. W. Aqueous-based extrusion fabrication of ceramics on demand. Eighteen Annual Solid Freeform Fabrication of Ceramic on Demand, Austin, TX, 2007; $124-133$.

18. Satish B, Ben C. Dynamic modeling and monitoring of contour crafting-an extrusion-based layered manufacturing process. Journal of Manufacturing Science and Engineering 2007; 129:135 - 142.

19. Liu G. A new low-priced rapid prototyping system. Technology and Innovation Conference, 2006. ITIC 2006. International, 2006; 856-860.

20. Silveira Z., de Freitas M. S., Inforçatti Neto P., Noritomi P. Y. , Silva J. V. Study of the technical feasibility and design of a mini head screw extruder applied to filament deposition in desktop 3-d printer. Key Engineering Materials, vol. 572, 2014; 151-154.

21. Freitas M. S., Silveira Z., Noritomi P., da Silva J., Rezende R. , et al.. Development of an innovative micro extrusion head based on screw for experimental additive manufacturing machines. Management and Control of Production and Logistics, vol. 6, 2013; 367-372.

22. Silveira Z., de Freitas M. S., Inforçatti Neto P., Noritomi P. Y. and da Silva J. V. L. Design development and functional validation of an interchangeable head based on mini screw extrusion applied in an experimental desktop 3-d printer. International Journal of Rapid Manufacturing 2014; 4(1):49-65.

23. Neto I, Noritomi P, Silva J, Freitas M, Silveira Z. Development of an interchangeable head based on variable section screw applied to desktop 3-d printers. High Value Manufacturing: Advanced Research in Virtual and Rapid Prototyping: Proceedings of the 6th International Conference on Advanced Research in Virtual and Rapid Prototyping, Leiria, Portugal, 1-5 October, 2013 2013; :19.

24. Turner B. N., Strong R. and Gold S. A. A review of melt extrusion additive manufacturing processes: I. process design and modeling. Rapid Prototyping Journal 2014; 20(3):192-204.

25. Kulshrestha M, Zaror C. An unsteady state model for twin screw extruders. Tran IChemE, PartC 1992; 70:21-28.

26. Li C. Modelling extrusion cooking. Food and Bioproducts Processing 1999; 77(1):55-63.

27. Diagne M, Couenne F, Maschke B. Mass transport equation with moving interface and its control as an input delay system. IFAC, 11th Workshop on Time-Delay Systems, WTC, Grenoble, France, vol. 11, 2013.

28. Diagne M. Modelling and control of systems of conservation laws with a moving interface: an application to an extrusion process. PhD Thesis, University Claude Bernard, Lyon 1, Lyon, France June 2013.

29. Diagne M., Krstic M. State-dependent input delay-compensated bang-bang control: Application to 3D printing based on screw extruder. Accepted ACC Chicago Illinois. 2015;

30. Diagne M, Shang P. and Wang Z. Feedback stabilization for the mass balance equations of an extrusion process. IEEE Transactions on Automatic Control 2015; :DOI:10.1109/TAC.2015.2444232.

31. Diagne M., Shang P. and Wang Z. Feedback stabilization of a food extrusion process described by 1d pdes defined on coupled time-varying spatial domains. 12th IFAC Workshop on Time Delay Systems, Ann Arbor, MI, USA, 2015.

32. Diagne M., Shang P., Wang Z. Cauchy problem for coupled hyperbolic systems through a moving interface. Mathematical Methods in the Applied Science (MMAS) (to appear) 2015; -(-):-.

33. Choulak S, Couenne F, Le Gorrec Y, Jallut C, Cassagnau P, Michel A. Generic dynamic model for simulation and control of reactive extrusion. Ind. Eng. Chem. Res 2004; 43:7373-7382.

34. Roberts S, Guy R. Instabilities in an extrusion-cooker: a simple model. Journal of Food Engineering 1986; 5(1):7 -30 .

35. Bekiaris-Liberis N., Krstic M. Compensation of state-dependent input delay for nonlinear systems. IEEE Transactions on Automatic Control 2013; 58(2):275-289.

36. Bekiaris-Liberis N, Krstic M. Robustness of nonlinear predictor feedback laws to time- and state-dependent delay perturbations. Automatica 2013; 49(4):1576-1590.

37. Bekiaris-Liberis N, Krstic M. Compensation of time-varying input and state delays for nonlinear systems. Journal of Dynamic Systems, Measurement, and Control 2012; 134(1):011 009.

38. Derezinski S. J. Calculating surge dampening in melt delivery systems. Society of Plastics Engineers Ann. Tech. Conf,Toronto 1997; .

39. Hatzikiriakos S. G. and Dealy J. M. Role of slip and fracture in the oscillating flow of hdpe in a capillary. Journal of Rheology 1992; 36(5):845-884.

40. Abeykoon C., McAfee M., Li K., Martin P. J., Kelly A. L. The inferential monitoring of screw load torque to predict process fluctuations in polymer extrusion. Journal of Materials Processing Technology 2011; 211(12):1907-1918.

41. Mudalamane R, Bigio D. I. Process variations and the transient behavior of extruders. AIChE Journal 2003; 49(12):3150-3160.

42. Tadmor Z, Lipshitz S. D. and Lavie R. Dynamic model of a plasticating extruder. Polymer Engineering \& Science 1974; 14(2):112-119, doi:10.1002/pen.760140206.

43. Kim E. K, White J. L. Isothermal transient startup for starved flow modular co-rotating twin screw extruder. Polymer Engineering and Science 2004; 40:543-553.

44. Kim E. K., White J. L. Non-isothermal transient startup for starved flow modular co-rotating twin screw extruder. International Polymer Processing 2004; 15:233-241.

45. Janssen L. P. B. M., Rozendal P. F., Hoogstraten H. W. and M. Cioffi. A dynamic model for multiple steady states in reactive extrusion. International Polymer Processing 2001; 16:263-271.

46. Janssen L. P. B. M., Rozendal P. F., Hoogstraten H. W. and M. Cioffi. A dynamic model accounting for oscillating behavior in extrusion reaction. International Polymer Processing 2003; 18:277-284.

47. Booy M. L. Isothermal flow of viscous liquids in corotating twin screw devices. Polymer Engineering Science 1980; 20:1220-1228. 
48. Booy M. L. Geometry of fully wiped twin-screw equipment. Polymer Engineering \& Science 1978; 18(12):973984.

49. Li C . Modelling extrusion cooking. Mathematical and Computer Modelling 2001; 33:553-563.

50. Fenner R. T., Cox A. P. D. and Isherwood D. P. Surging in screw-extruders. POLYMER 1979; 20:733-736.

51. Elsey J, Riepenhausen J, Mckay B, Barton G, Willis M. Modeling and control of a food extrusion process. Computers Chemical Engineering 1997; 21:361-366.

52. Kulshreshtha M., Zaror C. A, Jukes D. J. Simulating the performance of a control system for food extruders using model-based set-point adjustment. Food Control 1995; 6:135-141.

53. McAfee M, Thompson S. A novel approach to dynamic modeling of polymer extrusion for improved process control. Systems and Control Engineering 2007; 221:617-627.

54. Nield S, Budman H, Tzoganakis C. Control of a LPDE reactive extrusion process. Control Engineering Practice 2000; 8:911-920.

55. Moreira R, Srivastava A, Gerrish J. Feedforward control model for a twin-screw food extruder. Food Control July $1990 ; \mathbf{6}: 361-386$

56. Petit N. Control problems for one-dimensional fluids and reactive fluids with moving interfaces. Advances in the theory of control, signals and systems with physical modeling, Lecture notes in control and information sciences, vol. 407, Lausanne, 2010; 323-337.

57. Sontag E. On characterizations of the input-to-state stability property. Systems \& Control Letters 1995; 24(5):351359.

58. Krstic M. Input delay compensation for forward complete and strict-feedforward nonlinear systems. Automatic Control, IEEE Transactions on 2010; 55(2):287-303.

59. Karafyllis I, Krstic M. Numerical schemes for nonlinear predictor feedback. Mathematics of Control, Signals, and Systems $2014 ; 26(4): 519-546$.

60. Karafyllis I. Stabilization by means of approximate predictors for systems with delayed input. SIAM Journal on Control and Optimization 2011; 49(3):1100-1123.

61. Bresch-Pietri D., Leroy T., Chauvin J. and Petit N. Practical delay modeling of externally recirculated burned gas fraction for spark-ignited engines. Delay Systems, Advances in Delays and Dynamics, vol. 1. Springer International Publishing, 2014; 359-372.

62. L. Praly and Y. Wang, "Stabilization in spite of matched unmodeled dynamics and an equivalent definition of input-to-state stability," Mathematics of Control, Signals and Systems, vol. 9, no. 1, pp. 1-33, 1996.
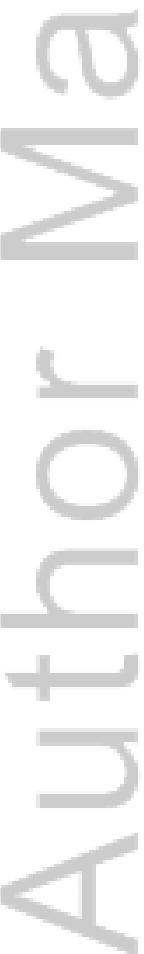


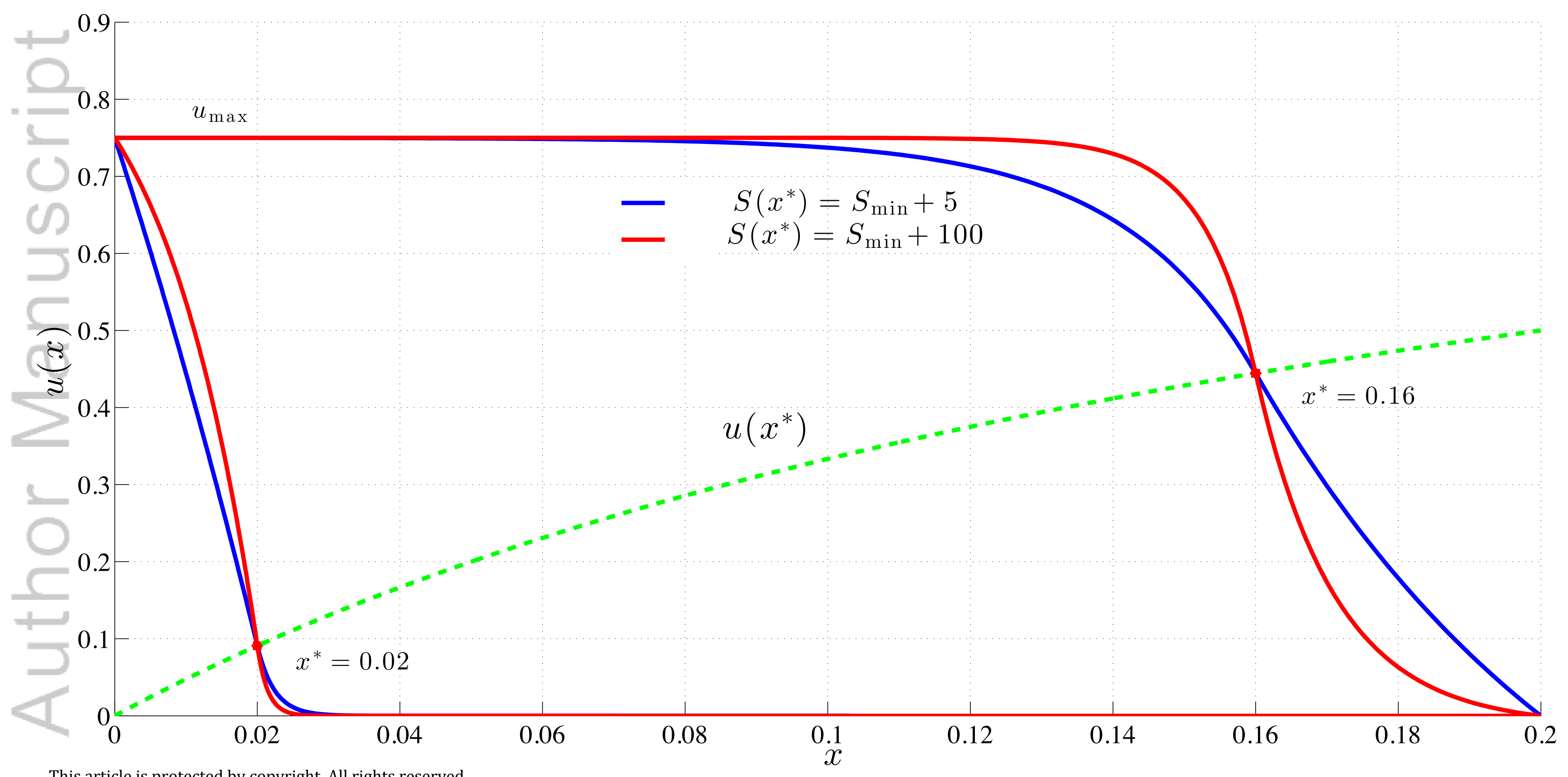

This article is protected by copyright. All rights reserved. 


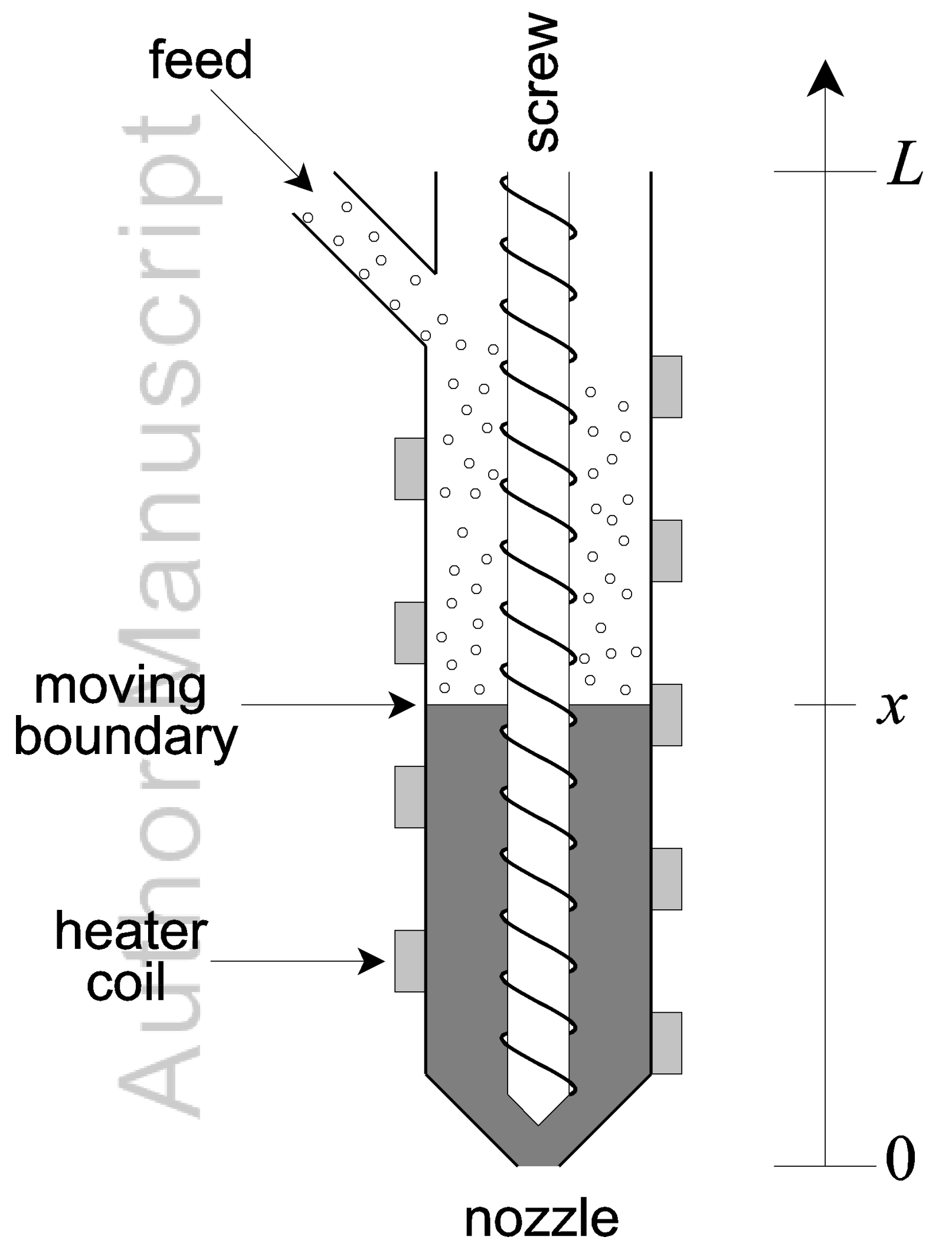

This article is protected by copyright. All rights reserved. 

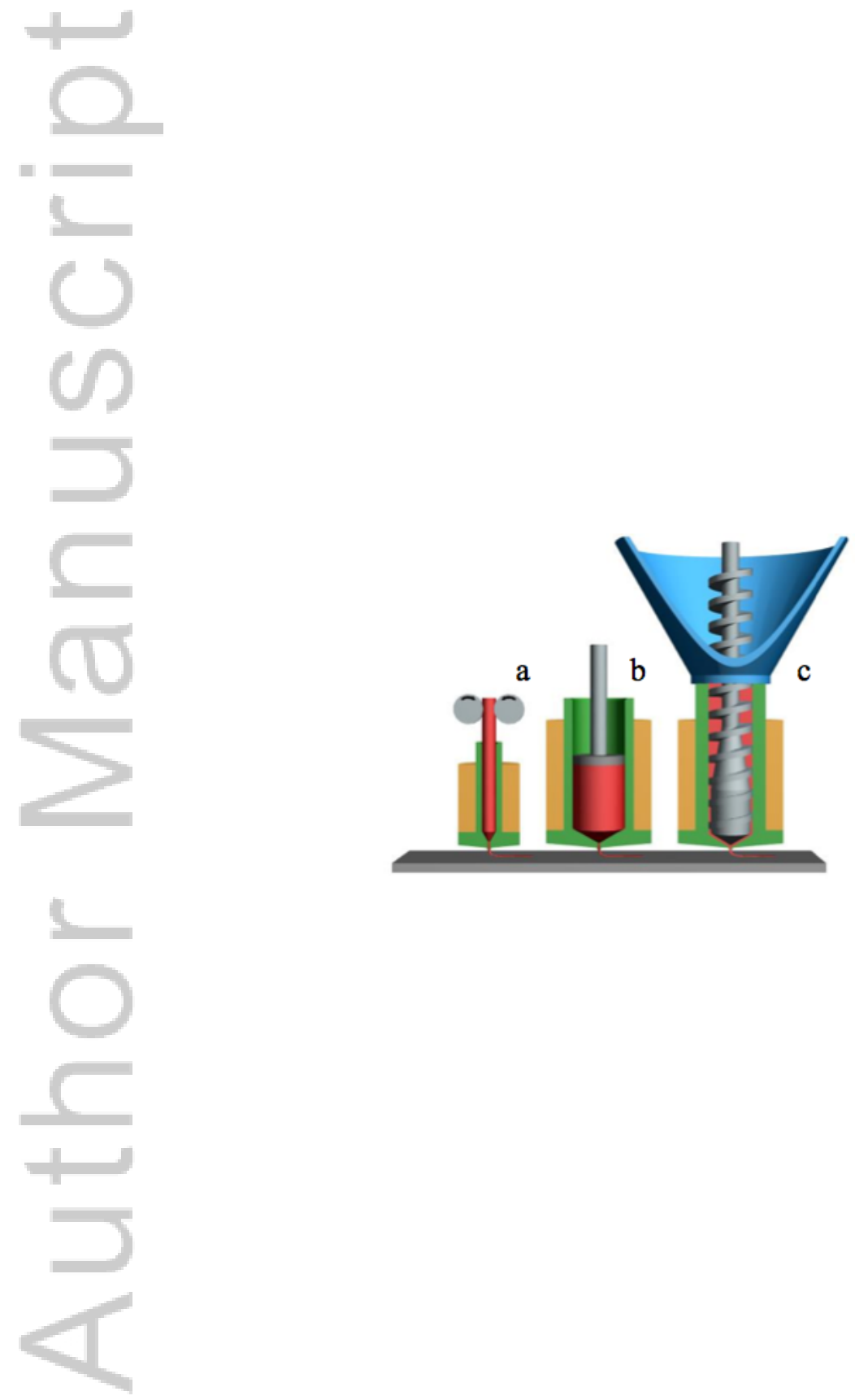

This article is protected by copyright. All rights reserved. 


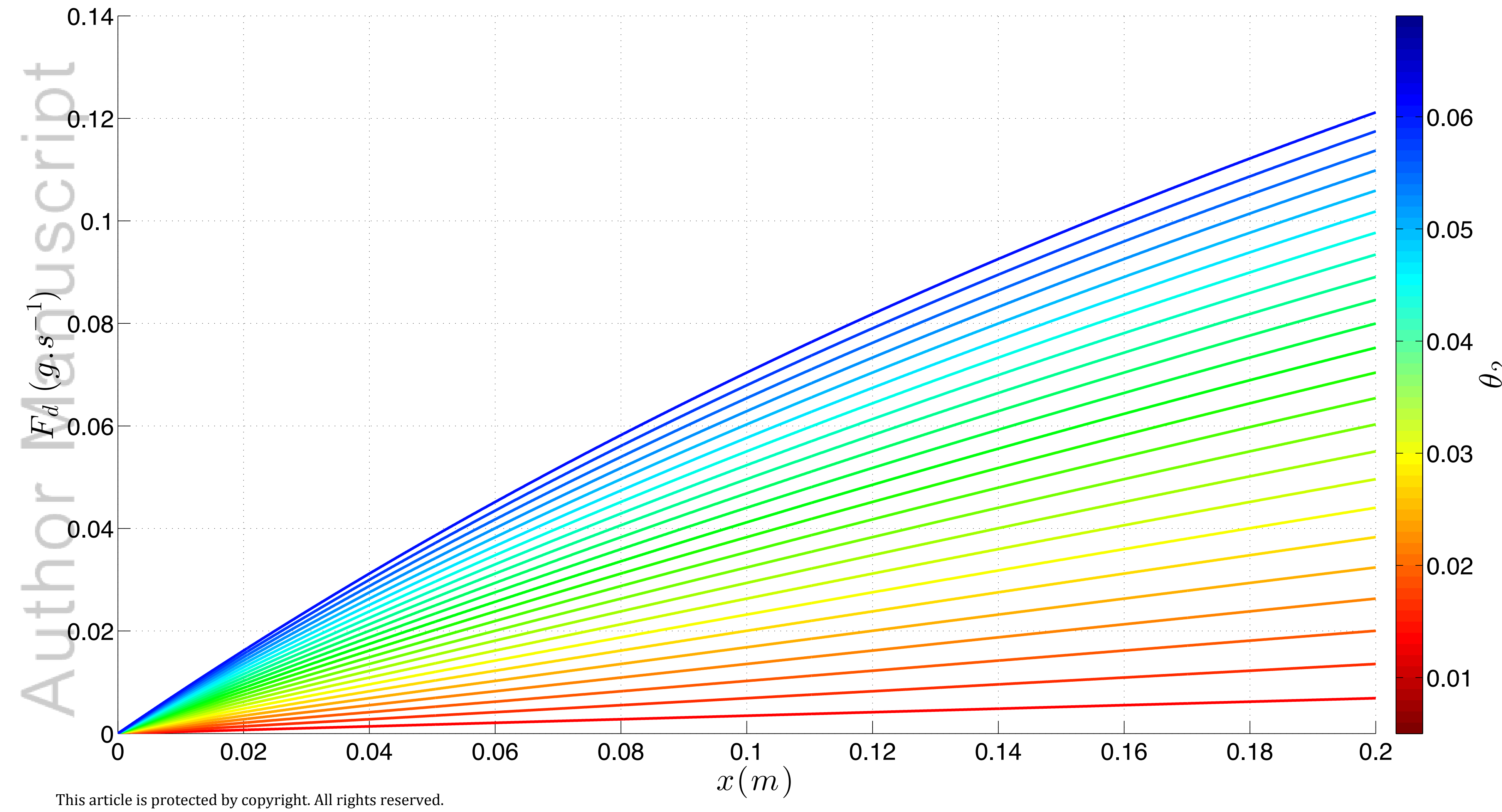

This article is protected by copyright. All rights reserved. 


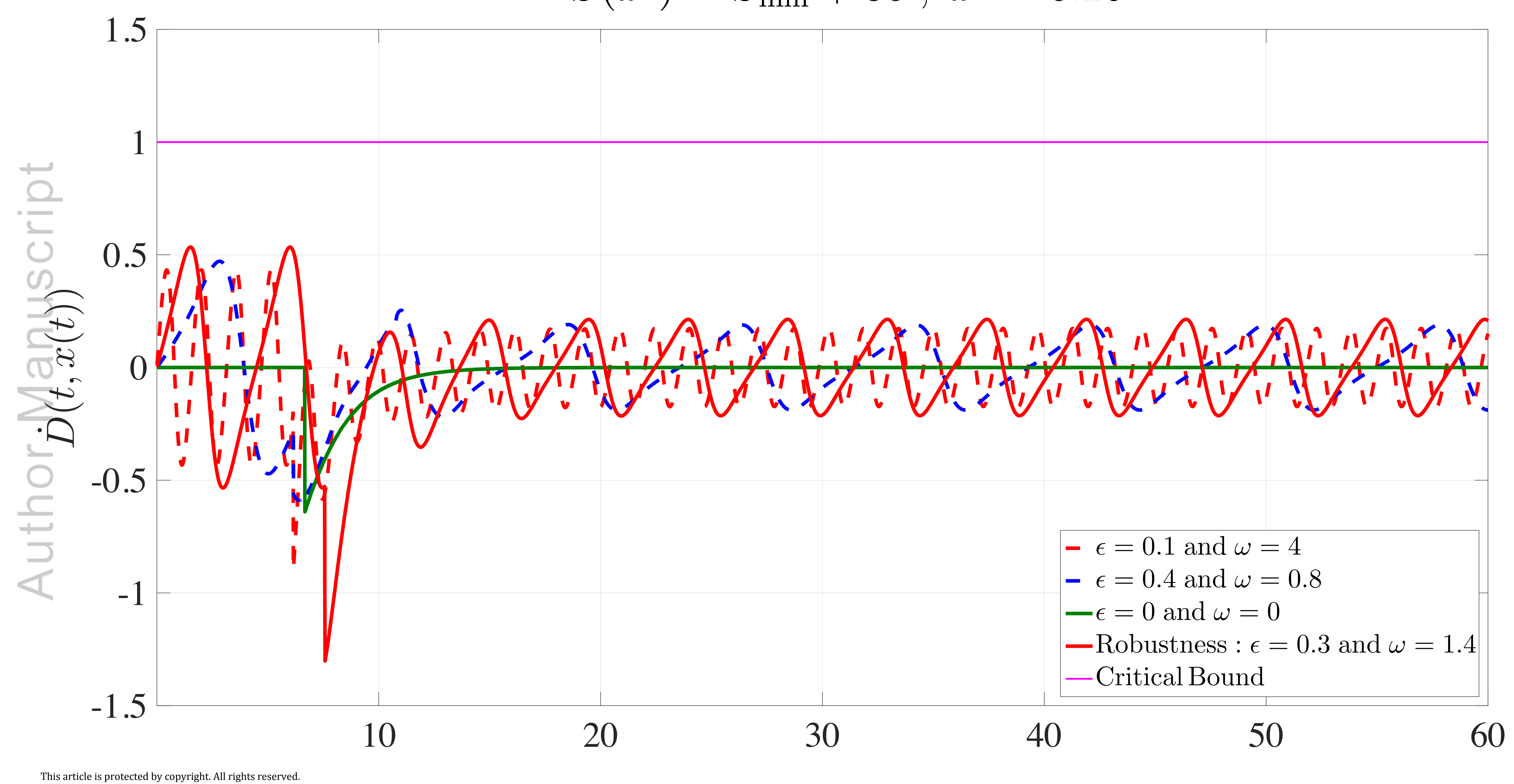


$S\left(x^{*}\right)=S_{\min }+30, x^{*}=0.16$

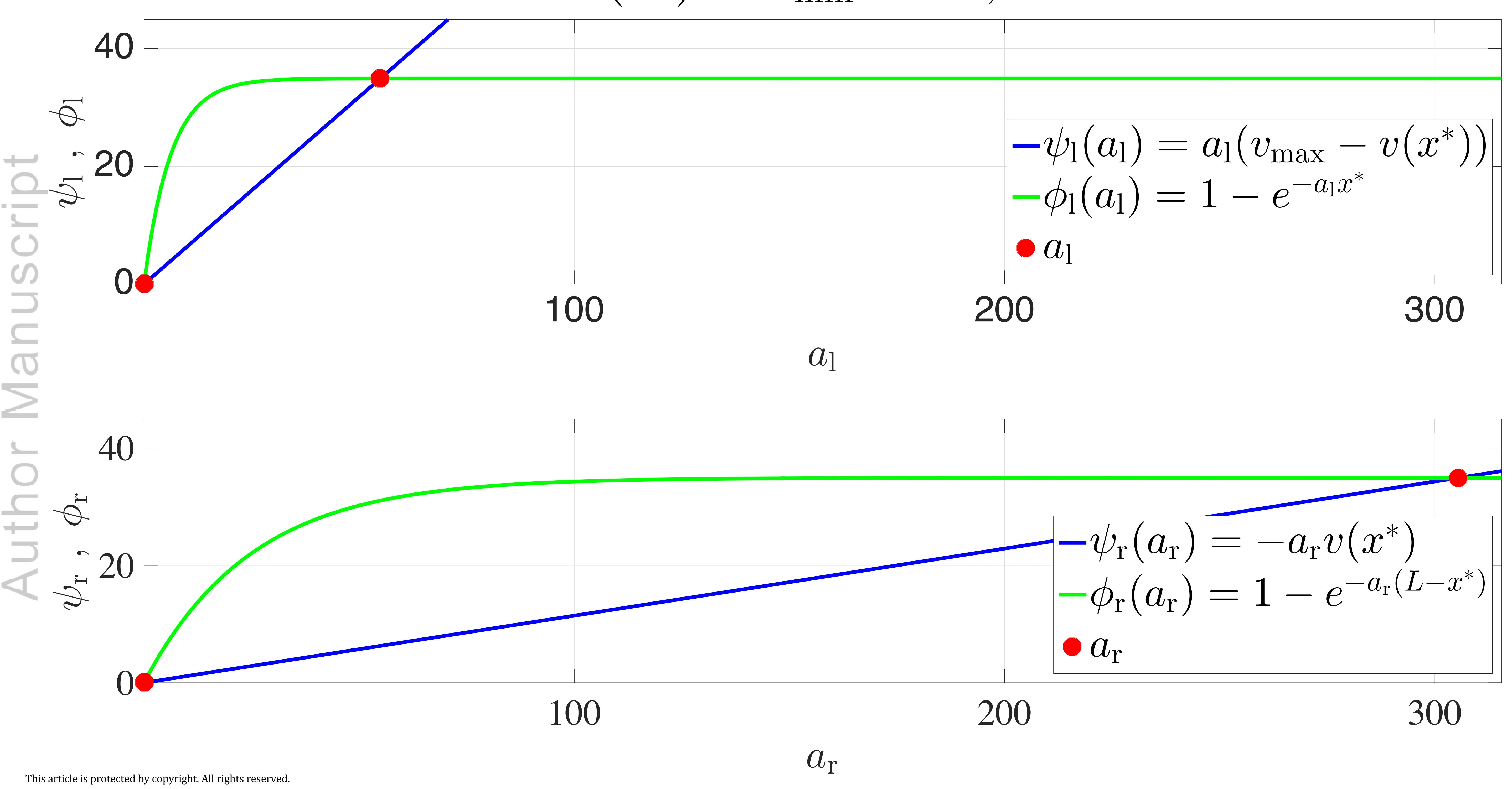


$0.2 \quad S\left(x^{*}\right)=S_{\min }+30, x^{*}=0.16, \epsilon=0.3, \omega=1.4$
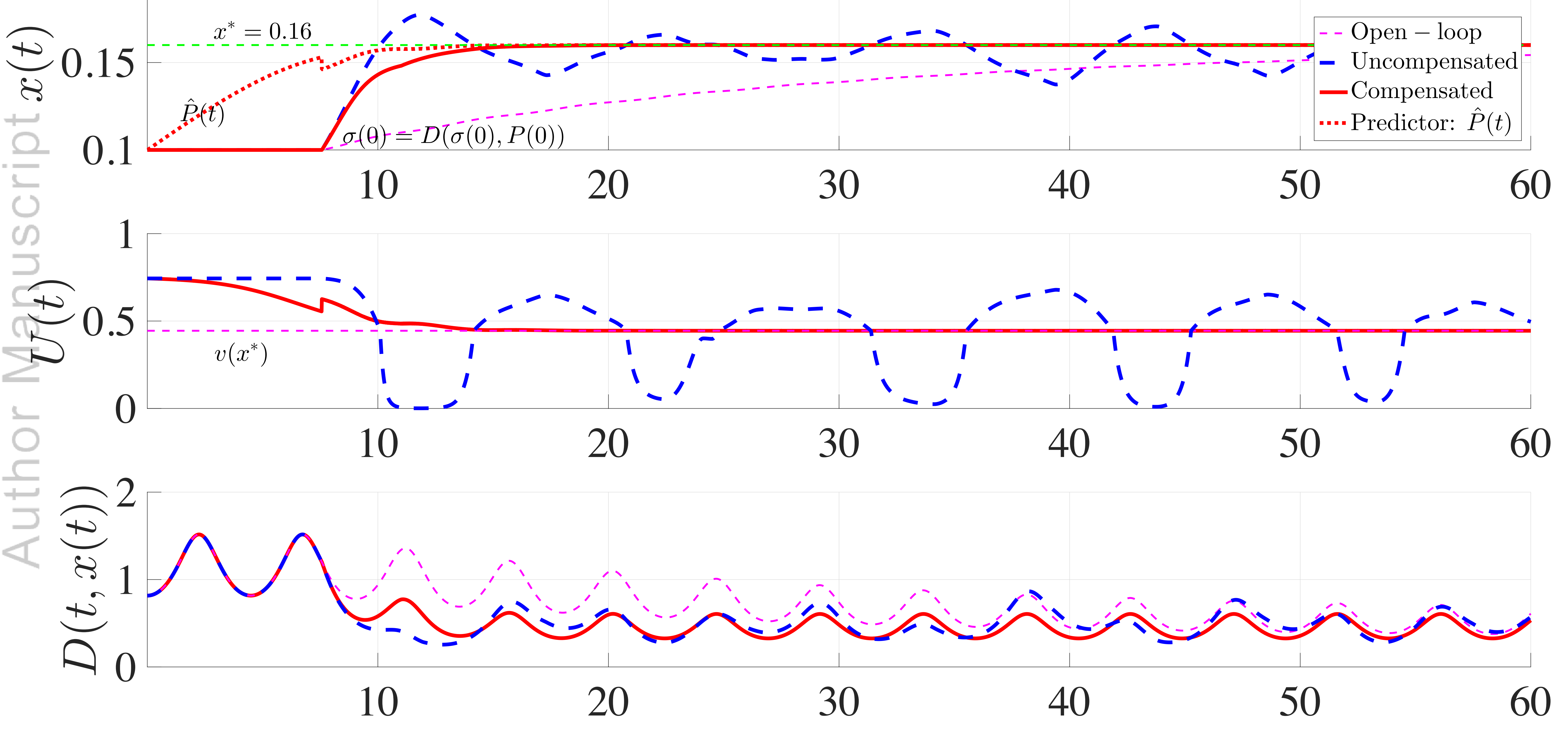
$S\left(x^{*}\right)=S_{\min }+30, x^{*}=0.16, \epsilon=0, \omega=0$

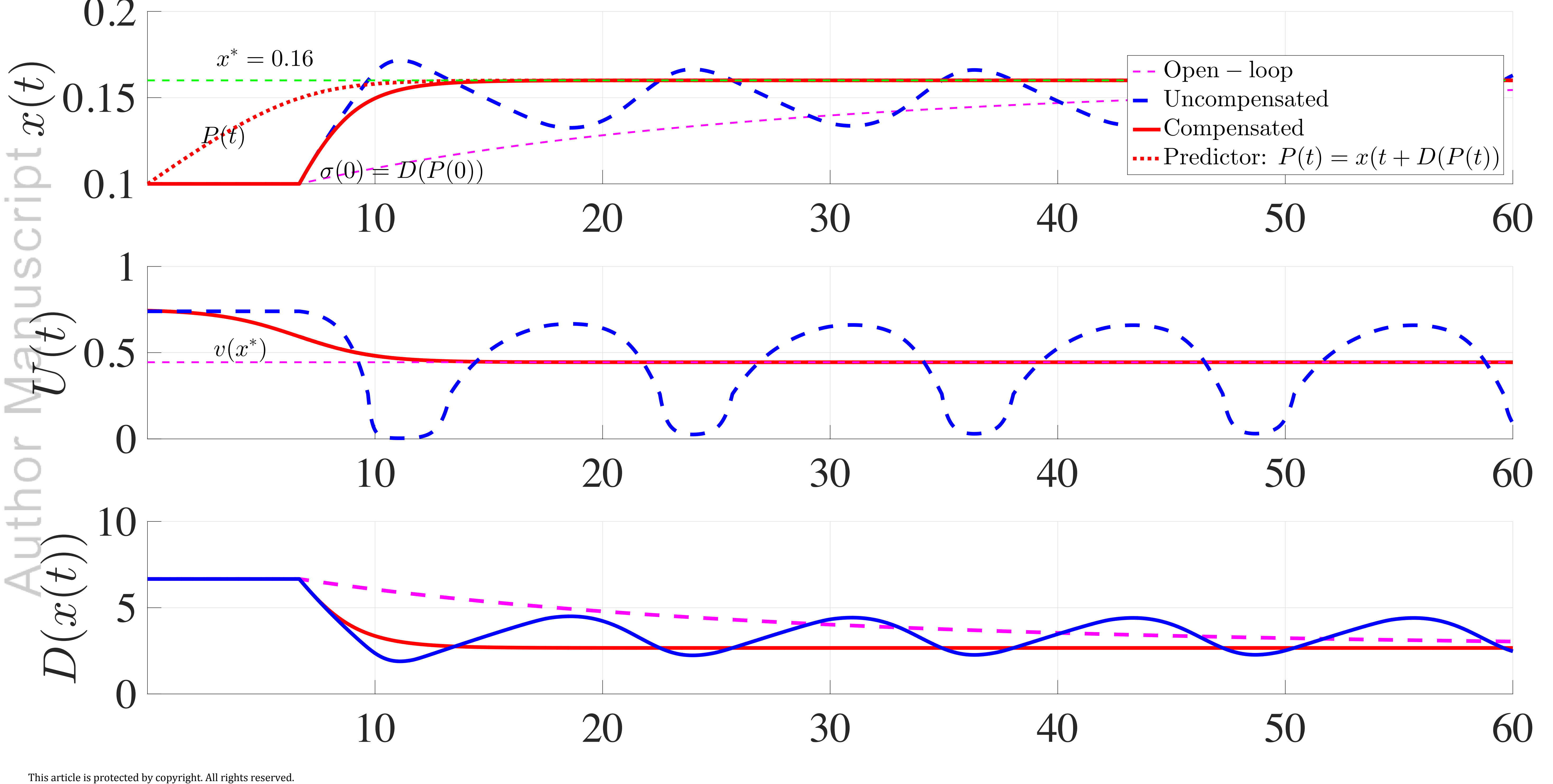


$0.2 \quad S\left(x^{*}\right)=S_{\min }+30, x^{*}=0.16, \epsilon=0.1, \omega=4$

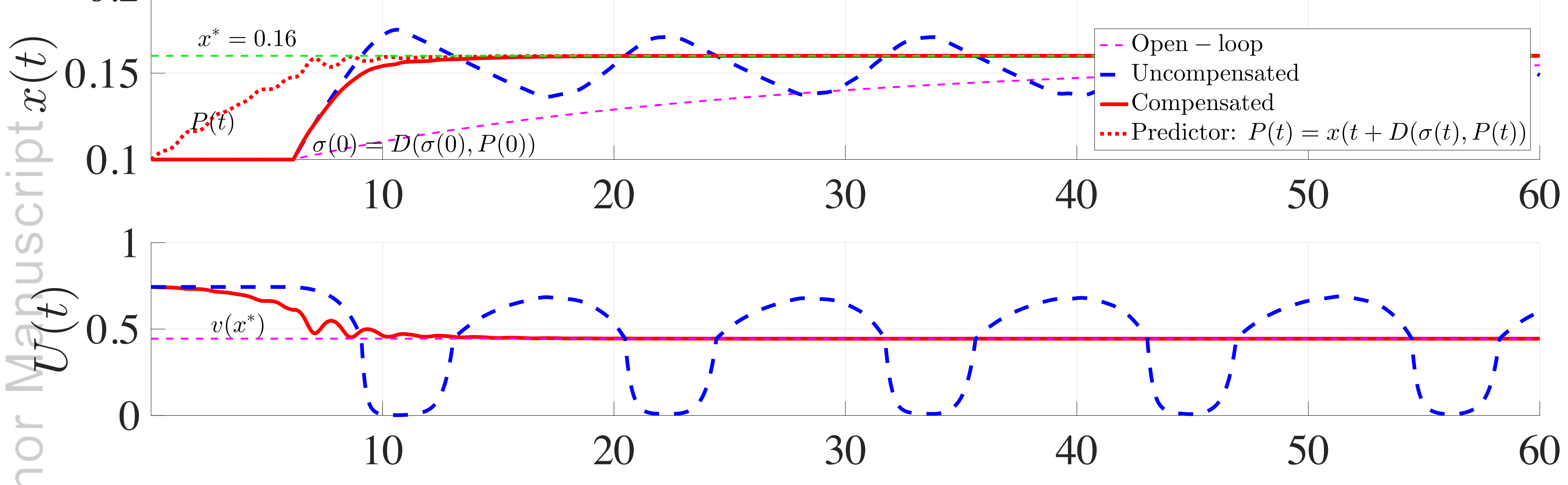

(ิ) 10

$\frac{\pi}{3}$
+

5

$w$

2

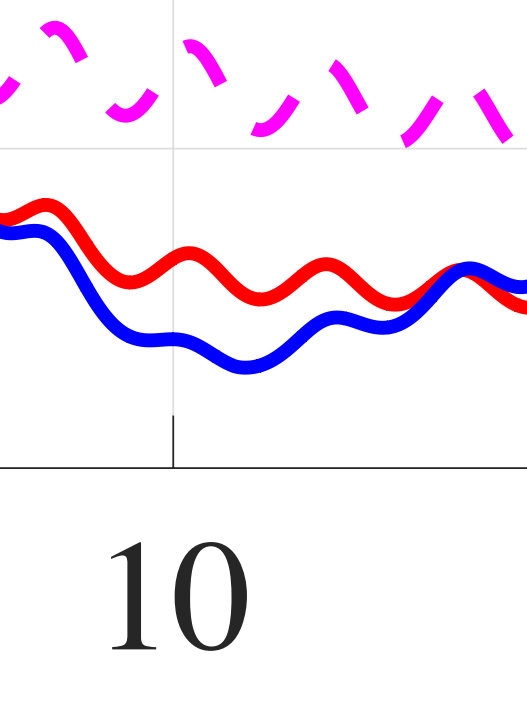

$\approx$

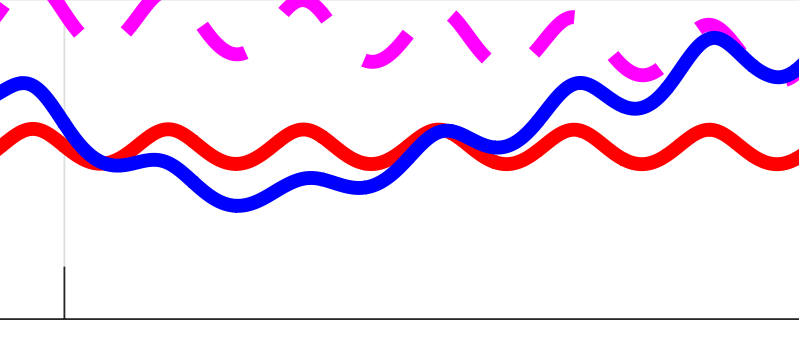


$0.2 \quad S\left(x^{*}\right)=S_{\min }+30, x^{*}=0.16, \epsilon=0.4, \omega=0.8$

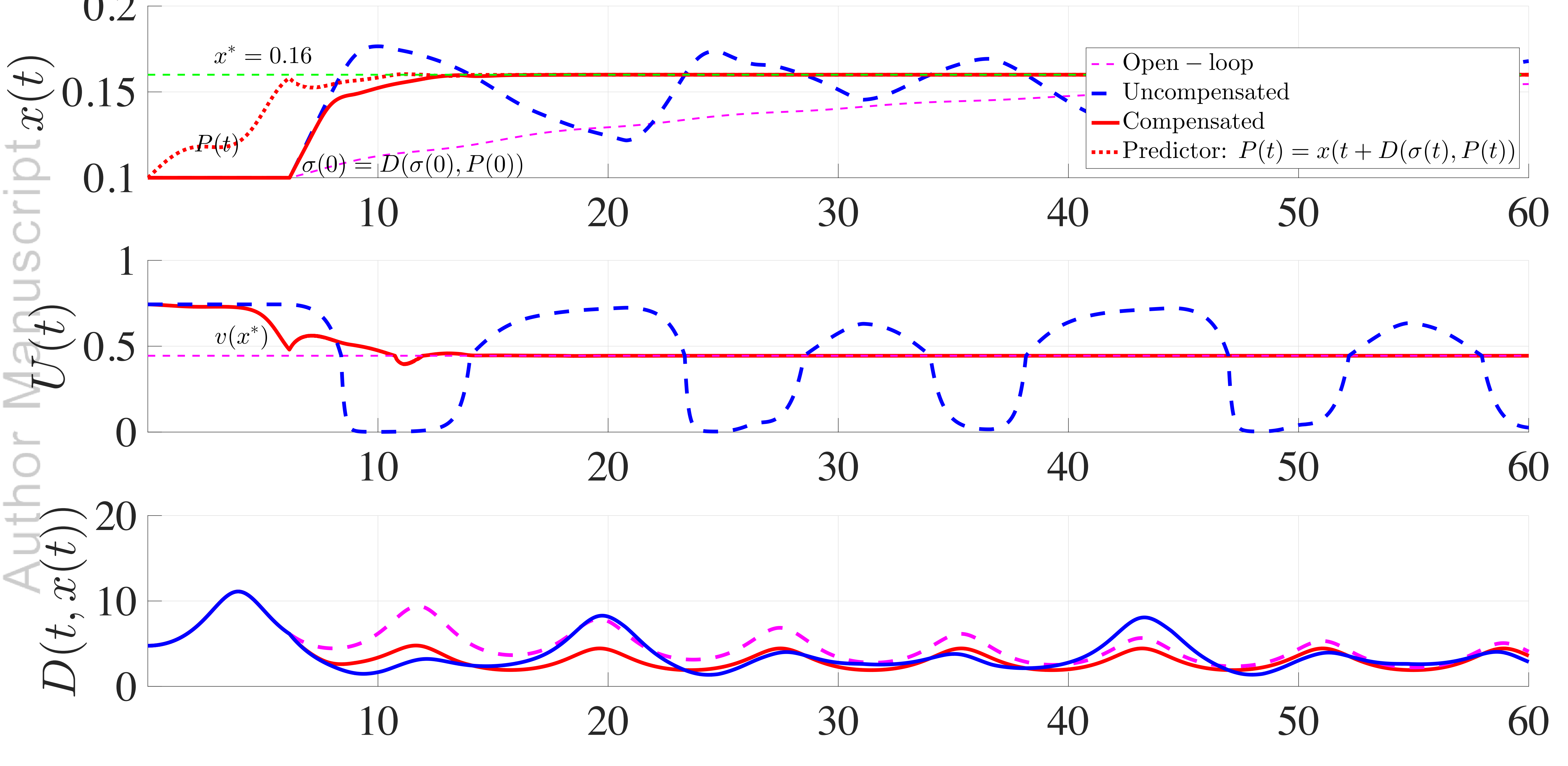

